\title{
6D fractional quantum Hall effect
}

\author{
Jonathan J. Heckman ${ }^{a}$ and Luigi Tizzano ${ }^{b}$ \\ ${ }^{a}$ Department of Physics and Astronomy, University of Pennsylvania, \\ Philadelphia, PA 19104, U.S.A. \\ ${ }^{b}$ Department of Physics and Astronomy, Uppsala University, \\ Box 516, SE-75120 Uppsala, Sweden \\ E-mail: jheckman@sas.upenn.edu, luigi.tizzano@physics.uu.se
}

ABSTRACT: We present a 6D generalization of the fractional quantum Hall effect involving membranes coupled to a three-form potential in the presence of a large background four-form flux. The low energy physics is governed by a bulk 7D topological field theory of abelian three-form potentials with a single derivative Chern-Simons-like action coupled to a $6 \mathrm{D}$ anti-chiral theory of Euclidean effective strings. We derive the fractional conductivity, and explain how continued fractions which figure prominently in the classification of $6 \mathrm{D}$ superconformal field theories correspond to a hierarchy of excited states. Using methods from conformal field theory we also compute the analog of the Laughlin wavefunction. Compactification of the 7D theory provides a uniform perspective on various lower-dimensional gapped systems coupled to boundary degrees of freedom. We also show that a supersymmetric version of the 7D theory embeds in M-theory, and can be decoupled from gravity. Encouraged by this, we present a conjecture in which IIB string theory is an edge mode of a 10+2-dimensional bulk topological theory, thus placing all twelve dimensions of F-theory on a physical footing.

Keywords: Chern-Simons Theories, M-Theory, Topological Field Theories, Topological States of Matter

ARXiv EPrint: 1708.02250 


\section{Contents}

1 Introduction $\quad 1$

$2 \quad 7 \mathrm{D}$ bulk 4

3 Many body wavefunction $\quad 9$

3.1 Landau wavefunction 11

$\begin{array}{ll}3.2 & \text { Zero slope limit of the membrane } \\ \end{array}$

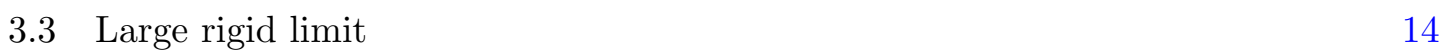

$\begin{array}{lll}3.4 & \text { Point particle limit } & 16\end{array}$

4 Quasi-branes and quasi-dranes $\quad 17$

$\begin{array}{llr}5 & \text { Compactification } & 19\end{array}$

5.1 Examples 20

6 Embedding in M-theory $\quad 22$

$\begin{array}{lll}7 & \text { IIB as an edge mode } & 25\end{array}$

8 Conclusions 31

\section{Introduction}

Extra dimensions provide a unifying perspective on a variety of lower-dimensional phenomena. This is by now quite commonplace in developing connections between the higherdimensional world of string theory and various low energy effective field theories. It also figures prominently in the analysis of many condensed matter systems, especially in the context of a bulk theory in a gapped phase coupled to boundary modes.

A classic example is the 2D fractional quantum Hall effect (FQHE) [1-6] and its connection with a bulk 2+1-dimensional Chern-Simons theory [7-15]. This involves an interplay between the theory of $2 \mathrm{D}$ chiral conformal field theories (CFTs), and a bulk topological field theory [16]. For a recent introduction to the subject see [17]. Various generalizations of this phenomena are by now available, including a higher-dimensional (integer) quantum Hall effect [18]. For additional discussion of the higher-dimensional quantum Hall effect in the condensed matter and string theory literature, see e.g. [19-25]. There is by now a vast literature on various ways that bulk gapped systems produce novel edge mode dynamics. ${ }^{1}$

\footnotetext{
${ }^{1}$ The list of such references is well-known to experts. For a review of some aspects of this and other condensed matter systems (with an eye towards holography) geared towards a high energy theory audience, see e.g. the review [26].
} 
From this perspective, it is natural to seek out additional examples of chiral conformal field theories as potential generalizations of the fractional quantum Hall effect to higher dimensions. Along these lines, there has recently been significant progress in understanding the construction of 6D supersymmetric conformal field theories (6D SCFTs). An important ingredient in these theories is that in the deformation away from the conformal fixed point, there are always anti-chiral two-form potentials with an anti-self-dual three-form field strength. This is a higher-dimensional analog of a chiral boson, and as such, there is no known way to write a Lorentz covariant action.

Our generalization of the fractional quantum Hall effect will involve a $6 \mathrm{D}$ anti-chiral theory of edge modes coupled to a $6+1$-dimensional bulk $M_{7}$ with Euclidean boundary $\partial M_{7}=M_{6}$. The 7D action we propose to study has been considered before in the high energy theory literature, both in the context of the topological sector of the AdS/CFT correspondence [27-29], and also as an interesting topological field theory in its own right [30-35]:

$$
S_{7 D}=\frac{\Omega_{I J}}{4 \pi i} \int_{M_{7}} c^{I} \wedge d c^{J},
$$

with $c^{I}$ a collection of three-form potentials with gauge redundancy $c^{I} \rightarrow c^{I}+d b^{I}$ and subject to the imaginary-self-dual boundary condition (see e.g. [31]):

$$
\left.*_{6 D} c^{I}\right|_{\partial M_{7}}=+\left.i c^{I}\right|_{\partial M_{7}} .
$$

Here, our sign convention is such that if we analytically continue to Lorentzian signature, the resulting boundary condition will yield an anti-self-dual three-form, in accord with the convention adopted for 6D SCFTs in references [36-38]. The pairing $\Omega_{I J}$ is an integral, positive definite symmetric matrix which is the analog of the " $K$-matrix" in the context of the standard fractional quantum Hall effect. The resulting 7D theory describes the low energy dynamics of $2+1$-dimensional membranes. In the boundary, these will appear as 2D Euclidean strings coupled to anti-chiral two-form potentials $b^{I}$ such that:

$$
c^{I}=d b^{I}
$$

In this context, the matrix $\Omega_{I J}$ governs the braiding statistics for Euclidean strings in the boundary $6 \mathrm{D}$ system. The existence of this bulk action is in some sense necessary to properly quantize the $6 \mathrm{D}$ theory since it is otherwise impossible to simultaneously impose a self-duality condition and quantization condition for three-form fluxes [31]. ${ }^{2}$

To realize both the integer and fractional quantum Hall effect, we activate a background magnetic four-form field strength, namely one in which all four legs thread the 6D boundary.

\footnotetext{
${ }^{2}$ The reason for this is that there is no integral basis of imaginary-self-dual three-forms on a generic sixmanifold with compact three-cycles. This is simply because the imaginary-self-duality condition depends on a choice of metric, and as such, continuous variation of the metric destroys the chance to have an integral basis of imaginary-self-dual three-forms. In the physical context, there is an additional caveat to this condition in the special case where $\operatorname{det} \Omega=1$ since then we have an invertible quantum field theory in the sense of reference [33]. In such situations one expects to be able to decouple the bulk and boundary theories since the boundary theory has a well-defined partition function.
} 
Using the 7D perspective, we argue that the many body wavefunction is constructed from correlation functions of non-local operators of the form:

$$
\Phi(\Sigma)=\exp \left(i m_{I} \int_{\Sigma} b^{I}\right),
$$

which from a 7D perspective involves replacing the integral of $b^{I}$ over a Riemann surface by an integral of $c^{I}$ over a three-chain with boundary $\Sigma$. Much as in the $2 \mathrm{D}$ case, our many body wavefunction factorizes as the product of a piece controlled by correlation functions involving the $\Phi$ 's, and a Landau wavefunction which dictates the overall size of droplets. Perhaps not surprisingly, this latter wavefunction is in turn controlled by the background four-form flux.

Though this correlation function is likely to be quite difficult to compute for an interacting $6 \mathrm{D} \mathrm{CFT}$, we find that in the case of a free theory of anti-chiral two-forms, the evaluation is relatively straightforward, and can be derived from general properties of conformal invariance. Said differently, the absence of a Lagrangian formulation for 6D chiral CFTs does not impede our analysis. The end result is somewhat more involved than that of the 2D Laughlin wavefunction, but even so, we find that it reduces to a quite similar form in a zero slope limit where the membranes are large and rigid. Other limits dictated by the relative energy scales set by the field strength and membrane tension lead to deviations from this simple behavior.

In the context of 6D CFTs with supersymmetry (which can be realized in a geometric phase of F-theory), the full list of $\Omega_{I J}$ has actually been classified [36-39]. From a geometric perspective, these matrices are nothing but the intersection pairings obtained from the resolution of orbifold singularities of the form $\mathbb{C}^{2} / \Gamma_{\mathrm{U}(2)}$ for $\Gamma_{\mathrm{U}(2)}$ a particular choice of discrete subgroup of $\mathrm{U}(2)$. The structure of this singularity is in turn controlled by continued fractions [40-42]:

$$
\frac{p}{q}=x_{1}-\frac{1}{x_{2}-\frac{1}{x_{3}-\ldots}} .
$$

From the perspective of the fractional quantum Hall effect, these are interpreted as filling fractions for excitations above the ground state [2-6].

The 7D starting point also provides a unifying perspective on a variety of lowerdimensional bulk topological systems coupled to dynamical edge modes. In these systems, the analog of the $K$-matrix is dictated by a tensor product $\Omega_{(7 D)} \otimes \Omega_{\text {(intersect) }}$, with $\Omega_{\text {(intersect) }}$ the intersection pairing on the compactification manifold. Indeed, even our 7D system can be viewed as the compactification of an 11D theory of five-forms placed on a background of the form $M_{7} \times M_{4}$.

The higher-dimensional unification in terms of this 11D theory suggests an irresistible further extension to twelve dimensions, especially in the context of F-theory, the nonperturbative formulation of IIB string theory. With this in mind, we present a speculative conjecture on what such a $12 \mathrm{D}$ theory ought to look like, showing that many elements can indeed be realized, albeit for a supersymmetric theory in $10+2$ dimensions. To avoid pathologies with having two temporal directions (such as moving along closed timelike 
curves), we demand from the start that our theory is purely topological in the bulk, namely the only propagating degrees of freedom are localized along a $9+1$-dimensional spacetime.

The rest of this paper is organized as follows. First, in section 2, we discuss in more detail the bulk 7D topological field theory which will form the starting point for our analysis. Using this perspective, we determine the associated fractional conductivities for membranes, and also present a formal answer for the analog of the Laughlin wavefunction. Next, in section 3 we evaluate the Laughlin wavefunction for a free theory of anti-chiral tensors. In section 4 we discuss the spectrum of quasi-excitations, and its interpretation in terms of an 11D topological field theory of five-forms. In section 5 we briefly discuss compactifications of the 7D (and 11D) bulk topological field theory to lower dimensions. Section 6 discusses the embedding of the 7D theory in M-theory, and section 7 presents our conjecture on F-theory as a $10+2$-dimensional topological theory. Sections 6 and 7 are likely to be of more interest to a high energy theory audience, and have been written so that they can be read independently of the other sections. We present our conclusions in section 8 .

\section{$2 \quad 7 \mathrm{D}$ bulk}

As mentioned in the introduction, our interest is in developing a higher-dimensional generalization of the fractional quantum Hall system. In this section, we lay out the main ingredients we use, focusing here on the 7D bulk description. Indeed, because we at present lack a microscopic description of $6 \mathrm{D}$ CFTs, it seems most fruitful to first develop the candidate effective field theory which would govern the low energy physics.

Our starting point is the $7 \mathrm{D}$ action: ${ }^{3}$

$$
S_{7 D}\left[c^{I}\right]=\frac{\Omega_{I J}}{4 \pi i} \int_{M_{7}} c^{I} \wedge d c^{J},
$$

in which we further assume that the seven-manifold is given by a product of the form:

$$
M_{7}=\mathbb{R}_{\text {time }} \times M_{6} .
$$

Said differently, when we proceed to quantize the theory, a wavefunction will involve coordinates defined on $M_{6}$, which we then evolve from one time slice to another.

There are various subtleties in quantizing such Chern-Simons-like theories, and we refer the interested reader to the careful discussion presented in references [31, 32, 44, 45].

\footnotetext{
${ }^{3}$ Following [29], in order to properly define (2.1), we need to specify the global nature of the fields $c$ 's and their gauge transformations. In this paper we assume that $c^{I} \sim c^{I}+Z_{2 \pi \mathbb{Z}}^{p}\left(M_{7}\right)$, where $Z_{2 \pi \mathbb{Z}}^{p}\left(M_{7}\right)$ is the space of closed $p$-forms with integral periods on $M_{7}$. To properly define this theory, it is helpful (much as in the ordinary Chern-Simons case) to view the 7-manifold as the boundary of an 8-manifold. More formally, one needs the analog of spin structure on a three-manifold, which is played in seven dimensions by a 4 -form $\mathrm{Wu}$ structure. This is necessary to have a properly defined theory of self-dual forms on the boundary. For further discussion on this and related points, see references [31, 43]. We shall neglect these subtleties in what follows since much of our analysis will focus on coarse features of the $6 \mathrm{D}$ fractional quantum Hall effect which can all be recast in terms of boundary correlators.
} 
Following the discussion in reference [27], we see that in a gauge where we set:

$$
\text { Coulomb Gauge: } c_{t a b}=0 \text {, }
$$

the canonical commutation relation for three-forms with all legs in spatial directions reads:

$$
\left[c_{a b c}^{I}(x), c_{d e f}^{J}(y)\right]=-2 \pi i \varepsilon_{a b c d e f}\left(\Omega^{-1}\right)^{I J} \delta^{6}(x-y),
$$

where $x$ and $y$ are coordinates on $M_{6}$. Note that to properly quantize the theory, we therefore need to specify a Lagrangian splitting of the phase space [31].

Much as in the case of 3D Chern-Simons theory, there is a class of observables of the form:

$$
\Phi_{m}(S)=\exp \left(i m_{I} \int_{S} c^{I}\right)
$$

for $S \in H_{3}\left(M_{6}, \mathbb{Z}\right)$, and $m_{I}$ a vector of charges. The pairing $\Omega_{I J}$ defines an integral lattice $\Lambda$, and the $m_{I}$ take values in its dual $\Lambda^{*}$.

The bulk correlation function of such observables is:

$$
\left\langle\Phi_{m_{1}}\left(S_{1}\right) \Phi_{m_{2}}\left(S_{2}\right) \ldots \Phi_{m_{N}}\left(S_{N}\right)\right\rangle_{7 D}=\exp \left[2 \pi i\left(m_{I}\left(\Omega^{-1}\right)^{I J} m_{J}\right) \sum_{1 \leq i<j \leq N} L\left(S_{i}, S_{j}\right)\right],
$$

where $L\left(S_{i}, S_{j}\right)$ is the integral linking number of $S_{i}$ and $S_{j}$. Given two bulk operators $\Phi_{m}(S)$ and $\Phi_{n}(T)$ for $m_{I}, n_{J} \in \Lambda^{*}$ and $S, T \in H_{3}\left(M_{6}, \mathbb{Z}\right)$, we get the braid relations [27]:

$$
\Phi_{m}(S) \Phi_{n}(T)=\Phi_{n}(T) \Phi_{m}(S) \times \exp \left(2 \pi i\left(m_{I}\left(\Omega^{-1}\right)^{I J} n_{J}\right)(S \cdot T)\right)
$$

where $S \cdot T$ is the intersection pairing for three-cycles in $M_{6}$.

The braiding relation of equation (2.7) tells us that in the quantum theory, we cannot simultaneously specify the periods of the three-form for all three-cycles in $M_{6}$. Rather, we must take a maximal sublattice of commuting periods and use this to specify the ground state(s). Calling this lattice of three-cycles $L \subset H_{3}\left(M_{6}, \mathbb{Z}\right)$, the degeneracy of the ground state is: ${ }^{4}$

$$
d_{\mathrm{GND}}=(\operatorname{det} \Omega)^{\operatorname{dim} L} .
$$

The three-form potential couples to $2+1$-dimensional membranes via integration over its worldvolume. These degrees of freedom are the analog of the electrons present in the fractional quantum Hall effect. In contrast to that case, however, there is no guarantee

\footnotetext{
${ }^{4} \mathrm{~A}$ more formal way to understand this relation is to note that states of the 7D bulk theory must assemble into representations of the Heisenberg group $\underline{H}^{3}\left(M_{6}, \Lambda^{*} / \Lambda\right)$. The maximal set of commuting fluxes $L \subset \underline{H}^{3}\left(M_{6}, \Lambda^{*} / \Lambda\right)$ leads to a ground state degeneracy of dimension $\left|\Lambda^{*} / \Lambda\right|^{\operatorname{dim} L}$. Note also that in the case of a 3D theory on $\mathbb{R} \times \Sigma$ for $\Sigma$ a genus $g$ Riemann surface, the degeneracy would be $(\operatorname{det} \Omega)^{g}$. Here we have neglected subtleties having to due with the presence of torsion. Some additional comments on the effects of this (which do not alter the degeneracy of the ground state) can be found in reference [35].
} 


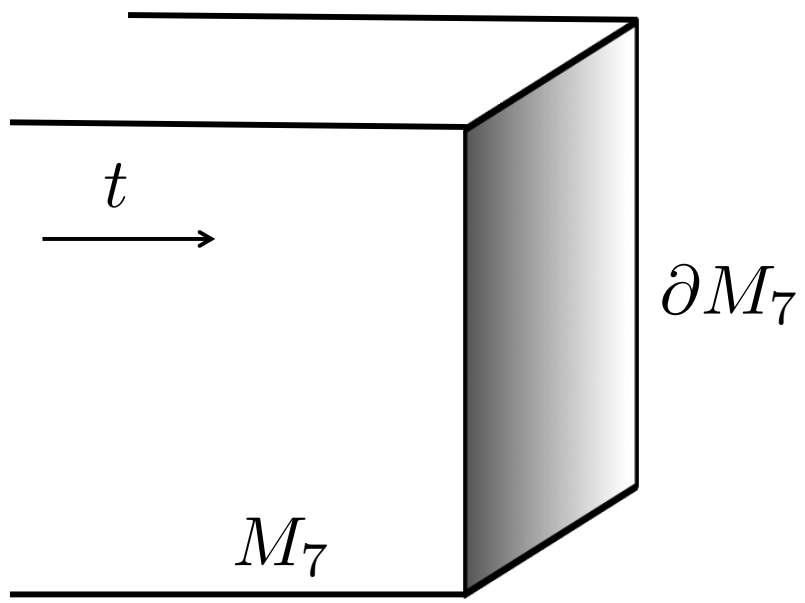

Figure 1. A representation of the bulk-boundary geometry considered in this paper. We denote the $7 \mathrm{D}$ bulk by $M_{7}$, the $6 \mathrm{D}$ boundary by $\partial M_{7}$ and time running into the boundary by $t$.

that these membranes are the genuine microscopic degrees of freedom for the 7D system. ${ }^{5}$ Indeed, from the perspective of M-theory, it is widely expected that M2-branes are also simply a collective excitation.

Suppose now that we consider our bulk theory on the spacetime

$$
M_{7}=\mathbb{R}_{\leq 0} \times M_{6},
$$

so that at $t=0$, we realize the boundary $\partial M_{7}=M_{6}$, namely we evolve a state from $t=-\infty$ to $t=0$. See figure 1 for a depiction of this geometry. In this case, we need to impose suitable boundary conditions for our system. Since we are assuming a Euclidean signature boundary manifold, we take:

$$
\left.*_{6 D} c^{I}\right|_{\partial M_{7}}=+\left.i c^{I}\right|_{\partial M_{7}},
$$

which if we analytically continue to $M_{6}$ a Lorentzian signature manifold (with mostly ${ }^{\prime}$ 's in the metric) would define an anti-self-duality relation.

From the perspective of the $6 \mathrm{D}$ boundary, we interpret the $c^{I}$ 's as three-form field strengths for two-forms $b^{I}$ :

$$
c^{I}=d b^{I}
$$

The two-form couples to a Euclidean effective string, namely the spatial section of our $2+1$-dimensional membrane. Including a membrane of charge $m_{I}$ at a particular time can therefore be described by inserting into the path integral the operator:

$$
\Phi_{m}(\Gamma)=\exp \left(i m_{I} \int_{\Gamma} c^{I}\right),
$$

\footnotetext{
${ }^{5}$ Moreover, there is a problem with formulating a theory of first quantized membranes as fundamental degrees of freedom as this generically introduces instabilities [46] (see also the review [47]). Here, however, there is not much of an issue since we are adopting an effective field theory perspective. Indeed, even if the membrane disintegrates, the macroscopic behavior we are considering should remain sensible since we are only interested in collective features.
} 


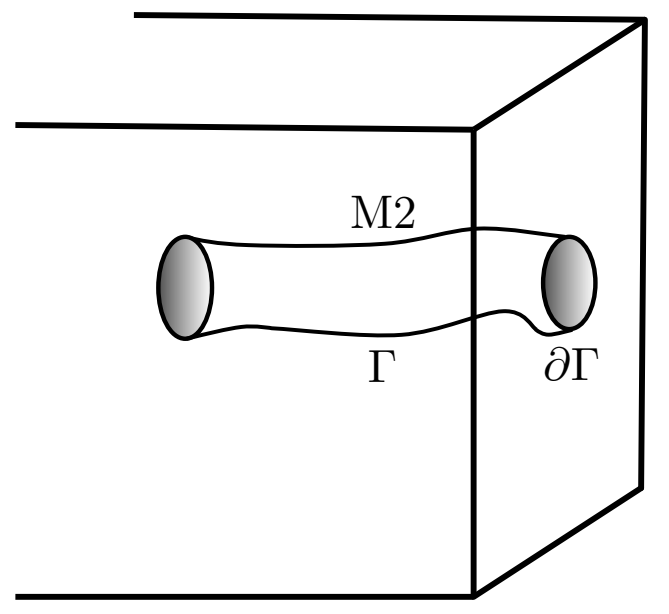

Figure 2. State insertion of a membrane wrapping a three-cycle $\Gamma$ with boundary $\partial \Gamma$.

where $\Gamma=\mathbb{R}_{\leq 0} \times \Sigma$ is a three-chain with boundary $\Sigma$ a Riemann surface wrapped by the membrane. See figure 2 .

The choice of charge vector $m_{I}$ for the membrane depends on whether we view it as a dynamic or static object in the $6 \mathrm{D}$ boundary theory. Indeed, though the $m_{I}$ take values in the dual lattice $\Lambda^{*}$, we can also entertain a special class of charges in the sublattice $\Lambda$ by instead working with $m_{I}=\Omega_{I J} n^{J}$ for $n^{J} \in \Lambda$. This distinction will prove important when we come to the structure of the Laughlin wavefunction where we will need to further restrict our choice of charges in this way.

A background collection of membranes is conveniently described by adding a source term $j_{I}$ to the action as follows:

$$
S_{7 D}\left[c^{I}, j\right]=\frac{\Omega_{I J}}{4 \pi i} \int_{M_{7}} c^{I} \wedge d c^{J}-i \int_{M_{7}} j_{I} \wedge c^{I} .
$$

The equations of motion in the presence of a background source are:

$$
j_{I}=\frac{\Omega_{I J}}{2 \pi} d c^{J}
$$

Adhering to the standard condensed matter terminology we dualize $j_{I}$ to a three-form. The two-point function for these three-index objects defines a six-index conductivity $\sigma_{a b c d e f}$, with all indices different.

Suppose now that we have a background three-form $C$ which couples to the membranes. In this interpretation, the $c^{I}$ simply correspond to various emergent gauge fields at low energies. The coupling between the two is:

$$
S_{7 D}\left[c^{I}, C\right]=\frac{\Omega_{I J}}{4 \pi i} \int_{M_{7}} c^{I} \wedge d c^{J}+\frac{\nu_{I}}{2 \pi i} \int_{M_{7}} C \wedge d c^{I} .
$$


Physically, the membranes are all charged under $C$, which has field strength $G$. In our conventions, the $G$-flux is quantized in units of $2 \pi:^{6}$

$$
\frac{1}{2 \pi} \int_{4 \text {-cycle }} G \in \mathbb{Z}
$$

Much as in the lower-dimensional setting, we then obtain a fractional conductivity given by:

$$
\sigma_{\text {abcdef }}=\frac{1}{2 \pi}\left(\Omega^{-1}\right)^{I J} \nu_{I} \nu_{J}
$$

Our discussion so far has focused on the bulk gapped system. Of course, it is also important to understand the structure of the boundary theory. We expect the many body wavefunction given by inserting a large number of membrane states in the presence of a background four-form flux to be in the same universality class as that of the genuine ground state. Each membrane corresponds to the insertion of an operator of the form given by line (2.12), for some choice of three-chain $\Gamma$ with boundary a Riemann surface $\Sigma$, and some choice of charge vector $m_{I}$ in the dual lattice of charges $\Lambda^{*}$. We label each such insertion in the path integral by an operator $\Phi^{(i)}$ for $i=1 \ldots, N$. The operator associated with a background four-form flux follows from line (2.15):

$$
\Phi_{\text {bkgnd }}=\exp \left(\frac{\nu_{I}}{2 \pi i} \int_{\mathbb{D}_{7}} c^{I} \wedge G\right)=\exp \left(\frac{\nu_{I}}{2 \pi i} \int_{\mathbb{D}_{6}} b^{I} \wedge G\right),
$$

where $\mathbb{D}_{7}=\mathbb{R}_{\leq 0} \times \mathbb{D}_{6}$ is a seven-dimensional domain, which at $t=0$ is given by a sixdimensional domain $\mathbb{D}_{6}$ which has no overlap with the locations of the Riemann surfaces $\Sigma_{(i)}$ wrapped by the membranes.

Putting all of this together, we expect the (unnormalized) many body wavefunction for $N$ such membranes to take the form:

$$
\Psi_{\text {Laughlin }}=\left\langle\Phi^{(1)} \ldots \Phi^{(N)} \Phi_{\text {bkgnd }}\right\rangle_{6 D},
$$

where the correlation function is evaluated in the boundary $6 \mathrm{D}$ theory.

Assuming that our $6 \mathrm{D}$ boundary theory is actually free, everything reduces to the calculation of appropriate two-point functions:

$$
\Psi_{\text {Laughlin }}=\prod_{1 \leq i<j \leq N}\left\langle\Phi^{(i)} \Phi^{(j)}\right\rangle_{6 D} \times \prod_{1 \leq i \leq N} \Psi_{\text {Landau }}^{(i)}
$$

where we have introduced the unnormalized Landau wavefunction for a single membrane moving in a background four-form flux:

$$
\Psi_{\text {Landau }}^{(i)}=\left\langle\Phi_{\text {bkgnd }} \Phi^{(i)}\right\rangle_{6 D}
$$

Our goal will be to estimate $\Psi_{\text {Laughlin }}$ in various limits.

\footnotetext{
${ }^{6}$ Strictly speaking, what is really required is that the integration over a difference of two four-cycles is integral. There can still be a half-integral shift, and this plays an important role in compactifications of M-theory [48].
} 


\section{Many body wavefunction}

In the previous section we presented a bulk perspective on the $6 \mathrm{D}$ fractional quantum Hall effect. Our aim in this section will be to extract additional details on the structure of the ground state wavefunction. To obtain concrete formulas, we focus on the case of a $6 \mathrm{D} \mathrm{CFT}$ in flat space, namely $\mathbb{R}^{6}$. Note that our result also generalizes to other conformally flat spaces such as $S^{6}$, or a $6 \mathrm{D}$ ball (namely the hyperbolic space $\mathbb{H}_{6}$ ). For ease of exposition, we shall focus on the theory of a single emergent three-form potential $c$, which couples to a uniform background magnetic four-form flux $G=d C$ (locally), so we consider:

$$
S_{7 D}[c, C]=\frac{\Omega}{4 \pi i} \int_{M_{7}} c \wedge d c+\frac{1}{2 \pi i} \int_{M_{7}} C \wedge d c .
$$

The generalization to the action of line (2.15) follows a similar line of argument.

The starting point for our analysis is the observation that on the $6 \mathrm{D}$ boundary, we have a theory of anti-chiral two-forms. In the case of a $2 \mathrm{D}$ chiral boson in flat space, there is a well-known (non-covariant) action given in reference [49]. In principle one could construct a similar action for the $6 \mathrm{D}$ anti-chiral two-form.

We shall not follow this route, but will instead simply appeal to the fact that we have a $6 \mathrm{D} \mathrm{CFT}$, and use the resulting structure of correlation functions for local operators. Indeed, we anticipate that our analysis will generalize to more involved interacting theories.

With this in mind, we need to extract the two-point function for non-local operators such as:

$$
\left\langle\Phi_{m}(\Sigma) \Phi_{m^{\prime}}\left(\Sigma^{\prime}\right)\right\rangle_{6 D}=\left\langle\exp \left(i m \int_{\Sigma} b\right) \exp \left(i m^{\prime} \int_{\Sigma^{\prime}} b^{\prime}\right)\right\rangle_{6 D},
$$

for some choice of charges $m$ and $m^{\prime}$, and Riemann surfaces $\Sigma$ and $\Sigma^{\prime}$ wrapped by the membranes. Here, the prime on $b^{\prime}$ serves to remind us that the potential is supported on $\Sigma^{\prime}$. For now, we treat these Riemann surfaces as fixed, though when we turn to the analysis of the Landau wavefunction, we will show how to fix their mean field values. Since we are dealing with a free field theory, we apply Wick's theorem to such correlation functions to write:

$$
\left\langle\Phi_{m}(\Sigma) \Phi_{m^{\prime}}\left(\Sigma^{\prime}\right)\right\rangle_{6 D}=\exp \left\langle-m m^{\prime} \int_{\Sigma \Sigma^{\prime}} b b^{\prime}\right\rangle_{6 D} .
$$

We therefore need to extract the integrated two-point function for the $b$-fields of our boundary theory. Strictly speaking, such a correlation function is not gauge invariant. Note, however, that by integrating over a closed Riemann surface, we should expect to obtain an answer independent of a particular gauge. Said differently, our operators and correlation functions are well-defined.

As we have already remarked, we do not have a covariant action for our anti-chiral two-form. Indeed, this is not even an operator in the 6D CFT. Rather, we know that the three-form field strength $h$ given locally by $d b$ is a well-defined operator. Our strategy will therefore be to compute the two-point function for $h$, and to then integrate this two-point function over a pair of three-chains with boundaries $\Sigma$ and $\Sigma^{\prime}$, respectively. 
The answer in this case follows directly from that given for a theory of two-forms with no self-duality constraint imposed. We follow the procedure outlined in references [50-53]. Denoting the field strength for this non-chiral two-form by $h_{n c}$, the two-point function is:

$$
\left\langle h_{a_{1} a_{2} a_{3}}^{(n c)}(x) h_{(n c)}^{b_{1} b_{2} b_{3}}(y)\right\rangle_{6 D}=\frac{18}{\pi^{3}} \times \frac{1}{r^{6}}\left[\delta_{\left[a_{1}\right.}^{b_{1}} \delta_{a_{2}}^{b_{2}} \delta_{\left.a_{3}\right]}^{b_{3}}-\frac{6 r_{\left[a_{1}\right.}{ }^{\left[b_{1}\right.} \delta_{a_{2}}^{b_{2}} \delta_{\left.a_{3}\right]}^{\left.b_{3}\right]}}{r^{2}}\right]
$$

where we have introduced the relative separation:

$$
r^{a}=x^{a}-y^{a} .
$$

Let us note that in this expression, there is in principle also a Dirac delta function contact term. This involves details about the microscopic theory, and can be removed by a suitable counterterm consistent with $6 \mathrm{D}$ conformal invariance. We note that this expression holds both in Minkowski and Euclidean spacetimes (by suitable choice of metric).

To reach the expression for the anti-chiral two-form theory, we apply a projection to imaginary-self-dual field strengths, namely we set:

$$
h=\frac{1}{2}\left(h_{(n c)}-i *_{6 D} h_{(n c)}\right) .
$$

The two-point function for the chiral theory is then:

$$
\left\langle h_{a_{1} a_{2} a_{3}}(x) h^{b_{1} b_{2} b_{3}}(y)\right\rangle=\frac{9}{\pi^{3}} \frac{1}{r^{8}}\left(r_{\left[a_{1}\right.} r^{\left[b_{1}\right.} \delta_{a_{2}}^{b_{2}} \delta_{\left.a_{3}\right]}^{\left.b_{3}\right]}+\frac{i}{6} r^{c} \varepsilon_{c a_{1} a_{2} a_{3}}^{\left[b_{1} b_{2}\right.} r^{\left.b_{3}\right]}\right),
$$

which is a somewhat more involved expression than its counterpart in two dimensions.

To obtain the integrated two-point function for the chiral two-forms, we now formally integrate this result over a pair of three-chains $\Gamma$ and $\Gamma^{\prime}$ inside of $\mathbb{R}^{6}$ :

$$
\left\langle\Phi_{m}(\Sigma) \Phi_{m^{\prime}}\left(\Sigma^{\prime}\right)\right\rangle_{6 D}=\exp \left\langle-m m^{\prime} \int_{\Gamma} \int_{\Gamma^{\prime}} h h^{\prime}\right\rangle_{6 D},
$$

where $\partial \Gamma=\Sigma$ and $\partial \Gamma^{\prime}=\Sigma^{\prime}$. Returning to equation (2.20), we see that our expression for the many body wavefunction:

$$
\Psi_{\text {Laughlin }}=\prod_{1 \leq i<j \leq N}\left\langle\Phi^{(i)} \Phi^{(j)}\right\rangle_{6 D} \times \prod_{1 \leq i \leq N} \Psi_{\text {Landau }}^{(i)}
$$

reduces to the calculation of these integrated two-point functions for the three-form fluxes.

So far, we have followed a quite similar plan to what is typically done in the $2 \mathrm{D}$ fractional quantum Hall effect; we have expressed the Laughlin wave function as a correlation function in a boundary CFT, and have also presented a formal expression for its structure (see e.g. [16] as well as the review in [17]).

But in contrast to the case of the 2D system which involves point particles, we are now faced with extended objects which carry an intrinsic tension:

$$
T_{M 2}=\frac{1}{\left(2 \pi \ell_{*}\right)^{3}} \text {. }
$$


Depending on the strength of the four-form flux, the membrane may either puff up to a large rigid object, or may instead be more accurately approximated by a point particle. This will in turn affect the shapes of the Riemann surfaces wrapped by the membranes.

We expect that a complete analysis will involve a generalization of the loop equations in QCD to the case of membranes, perhaps along the lines of references [51,54]. Even so, we can still use the structure of the correlation functions just extracted to approximate these dynamics. Our goal in the remainder of this section will be to characterize the typical size of a membrane, and to then use this to extract the behavior of the Laughlin wave function in various regimes. To this end, in the next subsection, we show that the Landau wavefunction factor always leads to a certain amount of "puffing up" for the membrane in all six spatial directions. This in turn depends on the particular profile for the four-form flux. After this, we turn to two special limits. In the limit where the membranes are very large compared to the intrinsic length scale $\ell_{*}$, we show that the Laughlin wavefunction actually reduces to a form quite close to that of the standard 2D Laughlin wavefunction. We also consider the opposite regime of dilute four-form flux in which the membranes are well-approximated by point particles.

\subsection{Landau wavefunction}

Our aim in this subsection will be to extract additional details on the structure of the Landau wavefunction factor appearing in equation (2.19), namely, the correlation function:

$$
\Psi_{\text {Landau }}=\left\langle\exp \left(\frac{\nu}{2 \pi i} \int_{\mathbb{D}_{6}} G \wedge b\right) \exp \left(i m \int_{\Sigma} b\right)\right\rangle_{6 D}=\left\langle\Phi_{\text {bkgnd }} \exp \left(i m \int_{\Sigma} b\right)\right\rangle_{6 D} .
$$

One of the implicit assumptions we have made up to this point is that the Riemann surface $\Sigma$ is held fixed. We now show that the presence of the four-form flux actually causes the membrane to puff up. As mentioned at the beginning of this section, we take the four-form flux to be uniform, and write:

$$
G=\frac{1}{4 !} G_{a b c d} d x^{a} \wedge d x^{b} \wedge d x^{c} \wedge d x^{d}
$$

where $x^{a}$ are local coordinates on $\mathbb{R}^{6}$ and we have taken some choice of constant $G_{a b c d}$. Suppose that we work in the limit where the Riemann surface $\Sigma$ is small. Then, we can parameterize its location, to leading order, by the position of the center of mass, which we denote by coordinates $y^{a}$. Applying the Laplacian in the $y$ variable to the correlation function yields:

$$
\Delta_{(y)}\left\langle\int_{\mathbb{D}_{6}} \frac{G}{2 \pi} \wedge b \int_{\Sigma} b^{\prime}\right\rangle_{6 D}=-\int_{\mathbb{D}_{6}} \frac{G}{2 \pi} \wedge *_{6 D} \delta_{\Sigma}^{(4)} .
$$

To reach the righthand side, we have used the fact that the membrane is - by definition localized along $\Sigma$, so we know that it has a delta function support for its source. Observe that in our integral, the only legs of $G$ which actually participate are those which are normal to the Riemann surface $\Sigma$. We denote these local coordinates by $y_{\perp}$. 


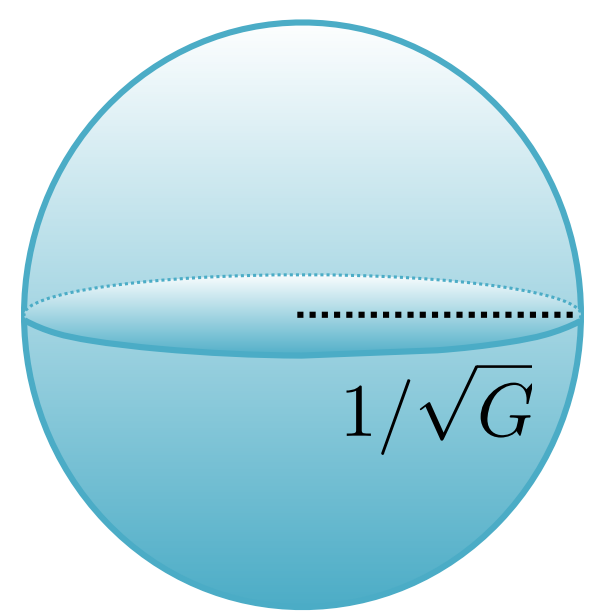

Figure 3. Depiction of a quantum Hall droplet of characteristic radius $\sim 1 / \sqrt{G}$.

On the other hand, we also know that if we simply consider the action of the Laplacian in these four directions, then we have:

$$
\Delta_{\perp} y_{\perp}^{2}=8
$$

Integrating equation (3.13), we conclude that our integral is, in this limit, given by a quadratic form in the normal directions:

$$
\left\langle\nu \int_{\mathbb{D}_{6}} \frac{G}{2 \pi} \wedge b \int_{\Sigma} b^{\prime}\right\rangle_{6 D}=-\frac{y_{\perp}^{2}}{8} \rho_{\perp},
$$

where we have introduced the localized flux density:

$$
\rho_{\perp}=\nu \int_{\mathbb{D}_{6}} \frac{G}{2 \pi} \wedge *_{6 D} \delta_{\Sigma}^{(4)} .
$$

At this point, we can now see why the presence of a background four-form flux prevents the membrane from collapsing to zero size. Indeed, from the above analysis, we see that if we consider the width of the membrane in the directions normal to $\Sigma$, there is a characteristic size $\ell_{\perp}$ indicating the spread of the associated Gaussian:

$$
\frac{1}{2 \ell_{\perp}^{2}} \sim \frac{G_{\perp}}{16 \pi}
$$

where $G_{\perp}$ denotes the component of the four-form flux with all legs transverse to $\Sigma$. Continuing in the same vein, we see that provided we have at least three independent contributions to the four-form flux, we always get a puffed up membrane. Said differently, there is no direction we can place a membrane so that it is not polarized.

It is helpful at this point to compare with the standard case of the $2 \mathrm{D}$ quantum Hall effect. There, we also have a droplet size, with characteristic length set by $\ell \sim 1 / \sqrt{F}$, in appropriate units. Here, we see the analog of this formula in equation (3.17). See also figure 3. In contrast to that case, however, we see that since the four-form flux always picks a preferred set of directions in the $6 \mathrm{D}$ space, we actually get a tensor of such characteristic lengths. 


\subsection{Zero slope limit of the membrane}

In the limit of a large magnetic flux, we also expect the worldvolume theory of the membrane to simplify. Here we present a brief sketch of how we expect such a simplification to occur. Some elements of our discussion are necessarily schematic, but we anticipate it will be useful for further investigations.

Consider the topological coupling between the three-form potential and the membrane:

$$
S_{\mathrm{M} 2} \supset \mu_{3} \int_{M_{3}} i^{*} C_{(3)},
$$

where $\mu_{3}$ is an overall constant of proportionality set by the tension of the membrane, $i$ : $M_{3} \rightarrow M_{7}$ is the embedding of the worldvolume of the membrane in the 7D spacetime, and $i^{*} C_{(3)}$ denotes the pullback of the bulk three-form onto the worldvolume. The embedding of the membrane is captured by fields $X^{a}\left(\sigma^{1}, \sigma^{2}, t\right)$. So, we can alternatively write the form of this coupling in components, where we absorb various numerical pre-factors into the overall coupling:

$$
S_{M 2} \supset \mu_{3} \int_{M_{3}} C_{a b c} d X^{a} \wedge d X^{b} \wedge d X^{c} .
$$

We are in particular interested in the special case of a large, uniform magnetic G-flux. In this limit, we anticipate that the action is actually dominated by this topological term. We refer to this as the zero-slope limit action:

$$
S_{\text {zero-slope }}=\mu_{3} \int_{M_{3}} G_{a b c d} X^{a} d X^{b} \wedge d X^{c} \wedge d X^{d} .
$$

The general algebraic structure associated with $C$ flat and large has been considered quite extensively in the literature (see e.g. [55-57] and references therein). As far as we are aware, however, the case of large G-flux has not been considered in much detail. Part of the complication with this sort of coupling is that it leads to a generalization of the Poisson bracket to a Nambu 2-bracket [58]. The quantum theory contains many technical complications, including the appearance of non-associative and non-commutative algebraic structures.

We bypass these complications (interesting though they may be) by focusing exclusively on the low energy limit, i.e. leading derivative contributions to the effective theory. This is accomplished most cleanly in the limit where we have a large background G-flux, since in this case, the membranes are polarized, and most fluctuations of the worldvolume will decouple.

Consider, for example, the expansion of the fields $X^{a}\left(\sigma^{1}, \sigma^{2}, t\right)$. In the limit of slow fluctuations, we can write:

$$
X^{a}\left(\sigma^{1}, \sigma^{2}, t\right)=X_{\mathrm{cm}}^{a}(t)+P_{\mathrm{cm}}^{a}(\sigma)+\ldots,
$$

where to leading order, the $X_{\mathrm{cm}}$ 's have no position dependence, and the $P_{\mathrm{cm}}$ 's have no time dependence. In this limit, then, we see that in the action of equation (3.20), each $X^{a}$ field can support at most one worldvolume derivative. Consequently, we can integrate by parts and present the action in the suggestive form:

$$
S_{\text {zero-slope }}=\mu_{3} \int_{M_{3}} G_{a b c d} \varepsilon^{\mu \nu \rho}\left(X^{[a} \partial_{\mu} X^{b]}\right) \partial_{\nu}\left(X^{[c} \partial_{\rho} X^{d]}\right) .
$$


Observe that the composite operators $X^{[a} \partial_{\mu} X^{b]}$ define a collection of abelian gauge fields on the three-dimensional space:

$$
Y_{\mu}^{a b}=X^{[a} \partial_{\mu} X^{b]}
$$

Interpreted in this way, we see that the G-flux can be split according to pairs of indices $a b$ and $c d$, and with respect to these indices, it is a symmetric matrix. Combining the parameter $\mu_{3}$ with that from $G$, we can now present the canonical form of the zero slope limit in terms of the gauge fields $Y_{\mu}^{a b}$ and a dimensionless matrix of couplings $K_{a b, c d}$ :

$$
S_{\text {zero-slope }}=\frac{K_{a b, c d}}{4 \pi i} \int_{M_{3}} Y^{a b} \wedge d Y^{c d} .
$$

Consider now the quantization of this worldvolume theory. In the gauge where $Y_{0}^{a b}=0$, the canonical commutation relation is:

$$
K_{a b, c d}\left[Y_{\mu}^{a b}(\sigma), Y_{\nu}^{c d}\left(\sigma^{\prime}\right)\right]=-2 \pi i \varepsilon_{\mu \nu} \delta^{2}\left(\sigma-\sigma^{\prime}\right) .
$$

The above commutation relation is the direct analog of what one finds for electrons moving in a large magnetic field. Indeed, in that context, the resulting 1D topological quantum mechanics is the starting point for the zero slope limit of open string theory in the presence of a large Neveu-Schwarz B-field [59]. It is quite tempting to also consider the limit of a large number of M2-branes, say in M-theory. The backreaction of the fourform flux on an individual membrane should then give, in a suitable limit, a Chern-Simons action. It would be interesting to connect these observations to the constructions presented in $[60,61]$.

\subsection{Large rigid limit}

Now that we have determined the impact of the four-form flux on the size of the droplets, we turn to the evaluation of the rest of the Laughlin wave function in line (2.20). This requires us to specify a pair of Riemann surfaces $\Sigma$ and $\Sigma^{\prime}$, as well as a pair of charges $m$ and $m^{\prime}$. In the limit where the background magnetic flux is very large, we see that the size of the membrane in the transverse directions is quite small, going roughly as $\ell_{\text {eff }} \sim 1 / \sqrt{G}$. In this limit, then, we approximate the membranes as wrapping very large Riemann surfaces (in units of the membrane tension length), and very thin in the transverse directions. To perform explicit computations, it is helpful to introduce a complex structure for $\mathbb{R}^{6}$, writing $\mathbb{C}^{3}$ with local coordinates $u, v, w$ such that the two Riemann surfaces are locally defined by the equations:

$$
\Sigma=\left\{u=u_{0}\right\} \cap\{w=0\} \quad \text { and } \quad \Sigma^{\prime}=\left\{u=u_{0}^{\prime}\right\} \cap\{v=0\}
$$

so that the common normal coordinate for both Riemann surfaces is parameterized by the $u$-plane, and the holomorphic coordinate:

$$
\xi=u_{0}-u_{0}^{\prime}
$$

specifies the separation between the two Riemann surfaces. 
To evaluate the correlation function of $\Phi(\Sigma)$ and $\Phi\left(\Sigma^{\prime}\right)$ in this limit, we observe that in the two-point function for the $b$ field, we are integrating (in momentum space) over the $w$ and $v$ directions. Consequently, we are actually working in the limit of low momentum in these two directions. The correlation function will therefore be dominated by momenta in the $u$-plane. With this in mind, we see that our problem reduces to a two-dimensional system.

In the related context where we compactify our $6 \mathrm{D}$ CFT on the space $\mathbb{R}^{2} \times \Sigma \times \Sigma^{\prime}$, the low energy theory on $\mathbb{R}^{2}$ is governed by a collection of chiral and anti-chiral bosons which all descend from the anti-chiral two-form. Indeed, we can decompose the $b$-field on shell as:

$$
b=\phi^{i}(\xi) \omega_{i}+\widetilde{\phi}^{\widetilde{i}}(\bar{\xi}) \omega_{\widetilde{i}}
$$

where $\omega_{i}$ is a basis of harmonic anti-self-dual two-forms on $\Sigma \times \Sigma^{\prime}$, and $\omega_{\widetilde{i}}$ is a basis of selfdual two-forms on $\Sigma \times \Sigma^{\prime}$. Here, the index $i=1, \ldots, b_{2}^{-}\left(\Sigma \times \Sigma^{\prime}\right)$ and $\widetilde{i}=1, \ldots, b_{2}^{+}\left(\Sigma \times \Sigma^{\prime}\right)$ so that the $\phi^{i}(\xi)$ are chiral bosons and the $\widetilde{\phi}^{\widetilde{i}}(\bar{\xi})$ are anti-chiral bosons. Integrating over the Riemann surfaces, we find that the correlation function reduces to:

$$
\left\langle\exp \left(i m \int_{\Sigma} b\right) \exp \left(i m^{\prime} \int_{\Sigma^{\prime}} b\right)\right\rangle_{2 D}=\xi^{\rho} \bar{\xi}^{\widetilde{\rho}}
$$

where in general the values of the $\rho$ and $\widetilde{\rho}$ depend on integrating the basis of self-dual and anti-self-dual forms over the Riemann surfaces. This in turn depends on the details of the metric. There is, however, an important aspect of this correlator which is protected by topology (see e.g. [62-65] ):

$$
\rho-\widetilde{\rho}=-\left(m \Omega^{-1} m^{\prime}\right)\left(\Sigma \cdot \Sigma^{\prime}\right)
$$

Note that single-valuedness of the associated OPE requires us to work in terms of charges $m$ and $m^{\prime}$ which scale in appropriate units of $\Omega$, as per our discussion below equation (2.12). The simplest possibility is to take $m=m^{\prime}=\Omega$, though more generally we can contemplate $m=n \Omega$ and $m^{\prime}=n^{\prime} \Omega$ so that the difference becomes:

$$
\rho-\widetilde{\rho}=-\left(n \Omega n^{\prime}\right)\left(\Sigma \cdot \Sigma^{\prime}\right)
$$

Returning to the evaluation of our correlation function, we see that there is a rather close similarity to the case of line (3.29). The main difference is that in the limit we have just taken, we have discarded various global data such as the topology of the Riemann surfaces. By construction, however, we have assumed that the only intersections occur when $\xi=0$. Consequently, we see that we can essentially carry over unchanged the calculation in the $2 \mathrm{D}$ limit. The precise values of the exponents $\rho$ and $\widetilde{\rho}$ also require information about the explicit choice of metric as well as the dynamics of the membranes moving in a background charge density. 
With this in place, we now generalize to other configurations of affine planes. It is helpful to introduce holomorphic homogeneous coordinates $Z^{\alpha}$ with $Z^{4}=1 .^{7}$ The Riemann surfaces can then be specified as:

$$
\begin{aligned}
\Sigma & =\left\{f_{\alpha} Z^{\alpha}=0\right\} \cap\left\{g_{\alpha} Z^{\alpha}=0\right\} \\
\Sigma^{\prime} & =\left\{f_{\alpha}^{\prime} Z^{\alpha}=0\right\} \cap\left\{g_{\alpha}^{\prime} Z^{\alpha}=0\right\} .
\end{aligned}
$$

In this case, the analog of the holomorphic separation between the two Riemann surfaces is now given by:

$$
\xi_{\Sigma, \Sigma^{\prime}}=\varepsilon^{\alpha \beta \gamma \delta} f_{\alpha} g_{\beta} f_{\gamma}^{\prime} g_{\delta}^{\prime},
$$

Assuming we remain in the rigid limit for all surfaces, we see that the resulting contribution to the Laughlin wavefunction in line (2.20) takes the form:

$$
\prod_{1 \leq i<j \leq N}\left\langle\Phi^{(i)} \Phi^{(j)}\right\rangle_{6 D} \sim \prod_{1 \leq i<j \leq N} \xi_{i j}^{\rho_{i j}} \widetilde{\xi}_{i j}^{\widetilde{\rho}_{i j}}
$$

in the obvious notation.

\subsection{Point particle limit}

We can also evaluate the form of the many body wavefunction in the limit where the relative separation between a pair of Riemann surfaces is quite large. In this case, the membranes are well-approximated by point particles moving in six spatial dimensions, and interacting via exchange of the bulk 7D three-form. We can therefore proceed in two complementary ways. On the one hand, we can simply calculate the scattering amplitude between two non-relativistic M2-branes. Alternatively, we can work directly in terms of the 6D CFT, and compute the long distance limit of the two-point function for the anti-chiral two-forms, suitably integrated over the small Riemann surfaces.

In either case, the problem reduces to that of a scattering amplitude as in figure 4 . We calculate the spin averaged value, neglecting issues of fine structure. Indeed, if we were to treat the Riemann surface as fixed, we would get a four-form source given by the corresponding delta function $\delta_{\Sigma}^{(4)}$, which dualizes to a two-form $\varepsilon_{c d}$ and couples to the $b$ field via $\varepsilon^{c d} b_{c d}$. At this point, it is convenient to work in Feynman gauge for the two-point function of the non-chiral two-form:

$$
\left\langle b_{a b}^{(\mathrm{nc})}(x) b_{c d}^{(\mathrm{nc})}(y)\right\rangle=\frac{1}{2 \pi^{3}} \frac{\eta_{a c} \eta_{b d}-\eta_{a d} \eta_{b c}}{(x-y)^{4}},
$$

The spin averaged amplitude is then given by:

$$
\mathcal{A}_{(\mathrm{nc})}=-\left(m_{(i)} \Omega^{-1} m_{(j)}\right) \times\left(\operatorname{Vol}\left(\Sigma_{(i)}\right) \times \operatorname{Vol}\left(\Sigma_{(j)}\right)\right) \times \frac{1}{\pi^{3}} \frac{1}{\left(x_{(i)}-x_{(j)}\right)^{4}} .
$$

\footnotetext{
${ }^{7} \mathrm{~A}$ curious feature of this formulation is the appearance of homogeneous coordinates, and therefore a $\mathbb{C P}^{3}$. Additionally, by specifying our Riemann surfaces by a pair of points in $\mathbb{C P}^{3}$, we also obtain the standard correspondence between twistor space and the complexification of conformally compactified four-dimensional Minkowski space [66]. The presence of a background four-form flux also suggests a noncommutative (possibly covariant) deformation of this space (see e.g. [67-69]). It would be interesting to develop a four-dimensional interpretation for the results of this paper.
} 


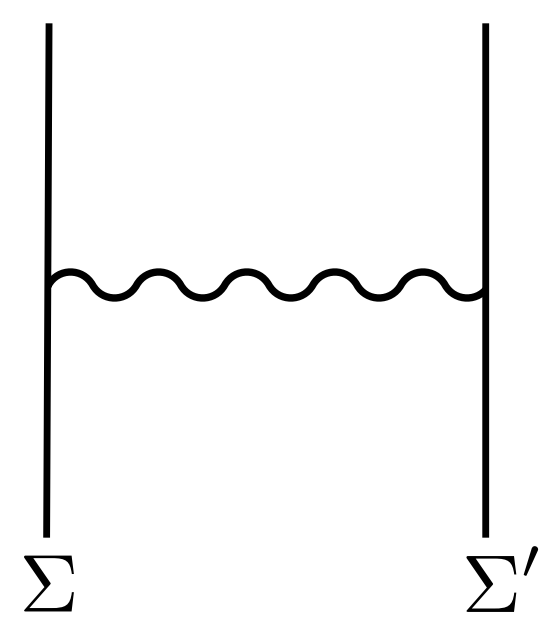

Figure 4. Exchange of an anti-chiral two-form between two membranes wrapped on Riemann surfaces $\Sigma$ and $\Sigma^{\prime}$.

Now, the amplitude receives two equal contributions, one from a basis of chiral twoforms and another from anti-chiral two-forms, with no cross-terms between the two. Because of this, the chiral case is half as large. Putting this together, we reach our estimate for the correlation function in this limit:

$$
\left\langle\Phi^{(i)} \Phi^{(j)}\right\rangle_{6 D}=\exp \left(\left(m_{(i)} \Omega^{-1} m_{(j)}\right) \times\left(\operatorname{Vol}\left(\Sigma_{(i)}\right) \times \operatorname{Vol}\left(\Sigma_{(j)}\right)\right) \times \frac{1}{2 \pi^{3}} \frac{1}{\left(x_{(i)}-x_{(j)}\right)^{4}}\right) .
$$

Observe that as we separate the particles, this correlator tends to one. In the opposite limit, the apparent divergence is cut off by the short distance behavior already described in the previous subsection. The crossover between these two regimes occurs precisely when the characteristic size of the Riemann surface becomes comparable to the separation.

\section{Quasi-branes and quasi-dranes}

As mentioned at the beginning of section 3, we can extend this analysis to more general pairings $\Omega_{I J}$. Indeed, there is a well-known interpretation of this in the standard fractional quantum Hall effect in terms of quasi-particle excitations/holes and their associated emergent gauge fields. To better understand this in our system, it is actually helpful to view the 7D bulk topological field theory as obtained from the compactification of an 11D theory of a single abelian five-form with action:

$$
S_{11 D}=\frac{1}{4 \pi i} \int_{M_{11}} C_{(5)} \wedge d C_{(5)} .
$$

Compactifying on a four-manifold, we assume the 11D spacetime takes the form of a Cartesian product $M_{11}=M_{7} \times M_{4}$. There is a decoupled sector given by a theory of threeforms. To study the structure of this subsystem, we can consider a basis of two-cycles $\Sigma_{I}$ with pairing $\Omega_{I J}=-\Sigma_{I} \cap \Sigma_{J}$. Dual to these cycles are harmonic two-forms $\omega^{I}$. To perform 
the reduction and maintain an integral basis of fields, it is actually most convenient to work in terms of the related basis of two-forms $\omega_{I}=\Omega_{I J} \omega^{J}$. Note that we also have:

$$
\Omega_{I J}=-\int_{M_{4}} \omega_{I} \wedge \omega_{J} .
$$

Expanding the five-form in terms of a basis of harmonic two-forms $\omega_{I}$ on $M_{4}$ yields:

$$
C_{(5)}=c_{(3)}^{I} \wedge \omega_{I}+\ldots,
$$

where the other terms in "..." refer to decoupled sectors. In this 11D theory, the degrees of freedom in the boundary are Euclidean D3-branes. These are wrapped over two-cycles of $M_{4}$, and this gives rise to the membranes discussed in the previous section. In this geometric construction, we take D3-branes wrapped over collapsing two-cycles. This leads to effective strings in six dimensions, which at the conformal fixed point have vanishing tension.

Indeed, in F-theory, the construction of 6D SCFTs involves compactification on a singular base $\mathcal{B}=\mathbb{C}^{2} / \Gamma_{\mathrm{U}(2)}$ with $\Gamma_{\mathrm{U}(2)}$ a discrete subgroup of $\mathrm{U}(2)$. Not all discrete subgroups realize a $6 \mathrm{D}$ SCFT, in part because they are incompatible with the existence of an elliptically fibered Calabi-Yau threefold with base $\mathcal{B}$. In the resolved phase, we have a generalization of Dynkin diagrams of ADE type. In fact, only the A- and D- series can have curves of self-intersection different from -2 . For additional details on the construction of 6D SCFTs in F-theory, see references [36, 38] as well as [70].

An intriguing feature of 6D SCFTs with an F-theory origin is the appearance of continued fractions such as:

$$
\frac{p}{q}=x_{1}-\frac{1}{x_{2}-\frac{1}{x_{3}-\ldots}} .
$$

As discussed in the introduction, the fractional quantum Hall effect also exhibits a sequence of continued fractions, and these numbers specify filling fractions for the spectrum of physical excitations above the ground state [2-6]. From an 11D perspective we can explain the appearance of this structure in terms of geometrical properties of the internal directions.

For example, in the generalization of an A-type base, this is a collection of curves of self-intersection $-x_{i}$ with pairing:

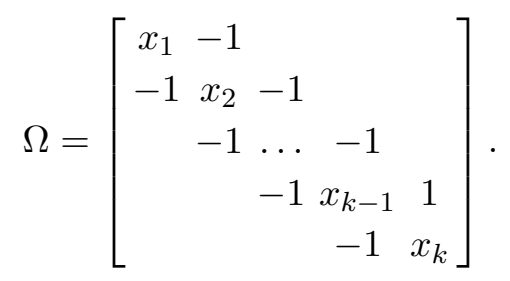

The orbifold singularity is then given by the group action on $\mathbb{C}^{2}$ with local coordinates $u$ and $v$ as:

$$
(u, v) \mapsto\left(\zeta u, \zeta^{q} v\right),
$$

for $\zeta$ a primitive $p^{\text {th }}$ root of unity (for example $\zeta=\exp (2 \pi i / p)$ ). 
Here we see that the spectrum of "quasi-particles" are actually Euclidean effective strings dictated by the continued fraction of line (4.4)! Moreover, we also know that at least for $6 \mathrm{D}$ SCFTs realized in F-theory, the space of possible pairings $\Omega_{I J}$ is tightly constrained. For example, the self-intersection numbers must always obey $1 \leq x_{i} \leq 12$. Additionally, further blowups of the base do not shift the value of $p$ or $q$, but do introduce additional quasi-branes [34, 71].

Turning the discussion around, it is natural to ask whether there is a top down interpretation of "quasi-dranes" (the negation of a brane). Physically, this would appear to descend from anti-Euclidean D3-branes. We leave an analysis of this issue for future work.

\section{Compactification}

One of the general paradigms of many condensed matter systems is the presence of a gapped bulk coupled to edge modes. It is also widely believed that this gapped phase is described by a topological field theory. Motivated by these considerations, in this section we use our higher-dimensional starting point as a tool in generating consistent examples of such phenomena. Our aim in this section will be to understand the class of theories generated from compactifications of our 7D bulk theory, and the corresponding edge modes. For simplicity, we focus on the case where there bulk flux is switched off.

In fact, following up on our discussion in section 4, it is helpful to actually begin with an 11D bulk theory of five-forms with action:

$$
S_{11 D}=\frac{1}{4 \pi i} \int_{M_{11}} C_{(5)} \wedge d C_{(5)} .
$$

Some aspects of this theory have been studied in [31,32]. Suppose now that we restrict the form of the $11 \mathrm{D}$ spacetime to be a product of the form:

$$
M_{11}=M_{11-p} \times M_{p}
$$

in which $M_{11-p}$ is a Lorentzian signature spacetime and $M_{p}$ is a Euclidean signature space. From the perspective of the boundary theory, we can take a limit in the space of metrics where $M_{p}$ is relatively small compared with $\partial M_{11-p}$. In this sense, we can "compactify" and reach a lower-dimensional theory defined solely on $M_{11-p}$.

To better understand the resulting theory, we decompose $C_{(5)}$ into a basis of harmonic differential forms defined on $M_{p}$ :

$$
C_{(5)}=\sum_{i=0}^{p} \sum_{k_{i}=1}^{b_{\mathrm{cpct}}^{i}\left(M_{p}\right)} C_{k_{i}}^{(5-i)} \wedge \omega_{k_{i}}^{(i)},
$$

namely, we sum over all compact harmonic $i$-forms with degeneracy label $k_{i}$, and also sum over all choices of $i$. Here, $b_{\mathrm{cpct}}^{i}\left(M_{p}\right)=\operatorname{dim} H_{\mathrm{DR}, \mathrm{cpct}}^{i}\left(M_{p}\right){ }^{8}$ There is a canonical pairing on

\footnotetext{
${ }^{8}$ Note that since we do not assume $M_{p}$ is compact, we cannot assert a relation between $b_{\text {cpct }}^{i}\left(M_{p}\right)$ and $b_{\mathrm{cpct}}^{p-i}\left(M_{p}\right)$.
} 
$M_{p}$ between an $i$-form $\omega_{k}$ and a $(p-i)$-form $\theta_{l}$ given by:

$$
\Omega_{k, l}^{(i),(p-i)}=\left\langle\omega_{k}, \theta_{l}\right\rangle=\int_{M_{p}} \omega_{k} \wedge \theta_{l}
$$

which defines a matrix of integers. The compactification of our 11D theory therefore reduces to a theory of abelian differential forms:

$$
S_{(11-p) D}=\sum_{i=0}^{p} \sum_{k_{i}=1}^{b_{\mathrm{cpct}}^{i}\left(M_{p}\right) \sum_{l_{i}=1}^{p-i}\left(M_{p}\right)} \frac{\Omega_{k_{i}, l_{i}}^{(i),(p-i)}}{4 \pi i} \int_{M_{11-p}} C_{k_{i}}^{(5-i)} \wedge d C_{l_{i}}^{(5-p+i)}
$$

Observe that this action breaks up into different non-interacting sectors. We always have a theory of $(5-i)$-forms coupled to $(5-p+i)$-forms, but these do not interact with the other sectors. ${ }^{9}$ For this reason, we can treat these contributions independently.

Note that we do not assume $M_{p}$ is compact. This means that the intersection pairings we generate, and thus the resulting matrix of couplings need not be square matrices, and when they are square, they need not have determinant one. For square matrices with $\operatorname{det} \Omega \neq 1$, the boundary theory is most appropriately viewed as a relative quantum field theory in the sense of reference [33] (see also [35]).

\subsection{Examples}

Let us give a few examples to show how we recover various topological field theories from this point of view. Isolating the contributions from the middle degree forms, we get the following bulk theories:

$$
\begin{aligned}
& S_{7 D}=\frac{\Omega_{I J}^{(7 D)}}{4 \pi i} \int C_{(3)}^{I} \wedge d C_{(3)}^{J} \\
& S_{6 D}=\frac{\Omega_{I J}^{(6 D)}}{2 \pi i} \int C_{(3)}^{I} \wedge d B_{(2)}^{J} \\
& S_{5 D}=\frac{\Omega_{I J}^{(5 D)}}{4 \pi i} \int B_{(2)}^{I} \wedge d B_{(2)}^{J} \\
& S_{4 D}=\frac{\Omega_{I J}^{(4 D)}}{2 \pi i} \int B_{(2)}^{I} \wedge d A_{(1)}^{J} \\
& S_{3 D}=\frac{\Omega_{I J}^{(3 D)}}{4 \pi i} \int A_{(1)}^{I} \wedge d A_{(1)}^{J} \\
& S_{2 D}=\frac{\Omega_{I J}^{(2 D)}}{2 \pi i} \int A_{(1)}^{I} \wedge d \phi_{(0)}^{J} \\
& S_{1 D}=\frac{\Omega_{I J}^{(1 D)}}{4 \pi i} \int \phi_{(0)}^{I} \wedge d \phi_{(0)}^{J},
\end{aligned}
$$

\footnotetext{
${ }^{9}$ In a theory with additional bulk matter fields, there can be one-loop induced mixing terms upon reduction to lower dimensions.
} 
in the obvious notation. There is a vast literature on nearly all of these theories, and so we shall limit our discussion to a few general comments.

These bulk topological field theories fall into two general subclasses, namely ChernSimons-like theories with potentials of the same degree and either symmetric or antisymmetric pairings, and BF-like theories with forms of different degree. In all of these cases, we expect to realize interesting edge mode dynamics, which in many cases can be understood from the compactification of a chiral four-form in ten dimensions to the lowerdimensional setting. Supersymmetry provides an additional extension of these results and leads to an even broader class of lower-dimensional theories.

One of the other lessons from string theory is that additional light degrees of freedom are expected to emerge in limits where the compactification manifold develops singularities. From this perspective, we can see that in many cases, we should expect to realize both additional non-abelian structure and higher spin currents in the boundary theory.

\subsubsection{BF-like theories}

We begin our discussion on effective topological field theories focusing first on dimensions 6, 4 and 2. Here, in general, we expect a BF-like theory as in equations (5.7), (5.9), (5.11).

Such BF theories feature prominently in long distance limits of various high energy physics systems and also play an important role in the description of gapped phases of matter. For example, a four-dimensional action similar to (5.9) was instrumental in the description of novel bosonic symmetry protected topological phases [72].

The 6D BF-like theory appears to have not received as much attention. Some details about this theory (and about BF theories of various dimensions) can be found in appendix A of [29]. It would be very interesting to determine the resulting theory of edge modes. It would also likely shed further light on the compactification of 6D SCFTs to five dimensions (see e.g. [73-75]).

\subsubsection{CS-like theories}

Consider next the Chern-Simons-like theories in which the differential forms all have the same degree. This occurs when the number of spacetime dimensions is odd.

The most familiar example in this class is given by abelian 3D Chern-Simons theory (5.10), which as we have already remarked is helpful in the study of the fractional quantum Hall effect $[9,13-15]$. Our interest in this paper has of course been the 7D generalization of this to three-forms.

Note that the theories in dimensions 7 and 3 have a symmetric matrix of couplings, as dictated by the intersection pairing on the internal space. By contrast, the theories in dimensions 5 and 1 have an anti-symmetric matrix of couplings, again in accord with the structure of the internal intersection pairing.

Finally, the 5D abelian Chern-Simons theory of two-forms in line (5.8) has appeared both in the condensed matter and high energy theory literature. For example, it appears in the low energy effective action of type IIB string theory on the background $\mathrm{AdS}_{5} \times S^{5}$ [27-29]. Alternative applications of line (5.8) concern the study of both gapped phases of matter [76] and discrete symmetries of gauge [77]. 


\section{Embedding in M-theory}

In our discussion up to this point, we have deliberately phrased our entire discussion in terms of a 7D topological field theory which is well-defined in its own right. It is nevertheless of interest to see how the 7D Chern-Simons-like theory we have been studying arises in compactifications of M-theory. An added benefit of this approach is that we will automatically show that there is a supersymmetric extension of our 7D theory.

Along these lines, we now take the 6D boundary theory to be a Lorentzian signature manfold, so that the $7 \mathrm{D}$ bulk coordinate is an additional spatial direction. Recall that we are interested in physical systems with interacting degrees of freedom localized along the $6 \mathrm{D}$ boundary which realize a chiral conformal field theory. At present, the only way to construct examples of such theories are supersymmetric and involve embedding in string/M-theory/F-theory, the first examples of this type being found in references [78-80].

A helpful example to keep in mind is the special case of M5-branes filling the first factor of $\mathbb{R}^{5,1} \times \mathbb{R}_{\perp} \times \mathbb{C}^{2} / \Gamma$ for $\Gamma$ a discrete subgroup of $\mathrm{SU}(2)$. We realize a conformal fixed point when the M5-branes all sit at the orbifold singularity of $\mathbb{C}^{2} / \Gamma$ and a common point of the $\mathbb{R}_{\perp}$ factor. This realizes the so-called class $\mathcal{S}_{\Gamma}$ conformal field theories studied in references $[37,81,82]$. We pass to the partial tensor branch of the theory where effective strings have a tension by moving the M5-branes apart from one another in the $\mathbb{R}_{\perp}$ direction. Observe that the geometry $\mathbb{R}^{5,1} \times \mathbb{R}_{\perp}$ is seven-dimensional, so it is natural to expect a bulk $7 \mathrm{D}$ theory to reside here which couples to the $6 \mathrm{D}$ boundary defined by the M5-branes.

Let us now turn to the construction and study of this putative 7D massive supermultiplet which contains a three-form potential and is decoupled from gravity. To see how this comes about, it is actually helpful to proceed up to eight dimensions, where supersymmetry has a chiral structure. Here, we have the following $8 \mathrm{D} \mathcal{N}=1$ massless supermultiplets (see e.g. [83, 84]):

8D Massless Supermultiplets:

$$
\begin{aligned}
G_{\mathcal{N}=1}^{8 D} & =1 \cdot[2]+1 \cdot\left[\frac{3}{2}\right]+2 \cdot[1]+1 \cdot\left[\frac{1}{2}\right]+1 \cdot[0]+1 \cdot\left[t_{2}\right] \\
S_{(3 / 2)}^{8 D} & =1 \cdot\left[\frac{3}{2}\right]+2 \cdot[1]+3 \cdot\left[\frac{1}{2}\right]+2 \cdot[0]+2 \cdot\left[t_{2}\right]+1 \cdot\left[t_{3}\right] \\
V_{(1)}^{8 D} & =1 \cdot[1]+1 \cdot\left[\frac{1}{2}\right]+2 \cdot[0]
\end{aligned}
$$

where the notation $[0],\left[\frac{1}{2}\right],[1],\left[\frac{3}{2}\right],[2]$ respectively refers to an $8 \mathrm{D}$ scalar, Weyl fermion, vector boson, gravitino and graviton. The notation $\left[t_{p}\right]$ refers to a $p$-form potential. We also have massive supermultiplets, where we denote massive fields by an overline:

8D Massive Supermultiplets:

$$
\begin{aligned}
\bar{S}_{(3 / 2)} & =1 \cdot \overline{\left[\frac{3}{2}\right]}+1 \cdot \overline{[1]}+2 \cdot \overline{\left[\frac{1}{2}\right]}+1 \cdot \overline{[0]}+1 \cdot \overline{\left[t_{2}\right]}+1 \cdot \overline{\left[t_{3}\right]} \\
\bar{V}_{(1)}^{8 D} & =1 \cdot \overline{[1]}+1 \cdot \overline{\left[\frac{1}{2}\right]}+1 \cdot \overline{[0]} .
\end{aligned}
$$


In terms of the field content, we have the following relations:

$$
\begin{aligned}
G_{\mathcal{N}=2}^{8 D} & =G_{\mathcal{N}=1}^{8 D}+S_{(3 / 2)}^{8 D}+2 \cdot V_{(1)}^{8 D} \\
\bar{S}_{(3 / 2)}^{8 D} & =S_{(3 / 2)}^{8 D} \\
{\overline{V^{8 D}}}_{(1)} & =V_{(1)}^{8 D} .
\end{aligned}
$$

The $8 \mathrm{D} \mathcal{N}=2$ multiplet $G_{\mathcal{N}=2}^{8 D}$ arises from M-theory compactified on a $T^{3}$.

Proceeding now to seven dimensions, we obtain the following irreducible 7D supermultiplets: ${ }^{10}$

$$
\begin{aligned}
G_{\mathcal{N}=1}^{7 D} & =1 \cdot[2]+2 \cdot\left[\frac{3}{2}\right]+4 \cdot[1]+4 \cdot\left[\frac{1}{2}\right]+4 \cdot[0]+1 \cdot\left[t_{2}\right] \\
S_{(3 / 2)}^{7 D} & =2 \cdot\left[\frac{3}{2}\right]+4 \cdot[1]+8 \cdot\left[\frac{1}{2}\right]+4 \cdot[0]+3 \cdot\left[t_{2}\right]+1 \cdot\left[t_{3}\right] \\
V_{(1)}^{7 D} & =1 \cdot[1]+2 \cdot\left[\frac{1}{2}\right]+3 \cdot[0] .
\end{aligned}
$$

The presence of the additional factor of two in the gravitino and Weyl spinors has to do with the way we count degrees of freedom for our spinors; in $8 \mathrm{D}$ we have a Weyl spinor, whereas in 7D we cannot have a Weyl spinor, and instead impose an appropriate reality condition.

Note that we have not "removed a vector multiplet" from the gravitino multiplet $S_{(3 / 2)}^{7 D}$. The reason this is not correct to do can be seen either by directly constructing the appropriate supermultiplet, or indirectly, by considering a further reduction to 6D, where removing such a multiplet would make it impossible to construct appropriate $6 \mathrm{D}$ supermultiplets. To see this explicitly, we decompose $S_{(3 / 2)}^{7 D}$ further into $6 D$ fields:

$$
\begin{aligned}
8 D & \rightarrow 7 D \rightarrow 6 D \\
S_{(3 / 2)}^{8 D} & \rightarrow S_{(3 / 2)}^{7 D} \rightarrow 2 \cdot\left[\frac{3}{2}\right]^{+}+2 \cdot\left[\frac{3}{2}\right]^{-}+10 \cdot\left[\frac{1}{2}\right]^{+}+10 \cdot\left[\frac{1}{2}\right]^{-}+8 \cdot[1]+8 \cdot[0]+4 \cdot\left[t_{2}\right] .
\end{aligned}
$$

And in six dimensions, we observe that we have the following $\mathcal{N}=(1,1)$ gravitino multiplets:

$6 D$ Gravitino Supermultiplets:

$$
\begin{aligned}
& S_{(3 / 2)}^{+}=\left[\frac{3}{2}\right]^{+}+2 \cdot[1]+4 \cdot\left[\frac{1}{2}\right]^{+}+1 \cdot\left[\frac{1}{2}\right]^{-}+2 \cdot[0]+2 \cdot\left[t_{2}\right]^{+} \\
& S_{(3 / 2)}^{-}=\left[\frac{3}{2}\right]^{-}+2 \cdot[1]+4 \cdot\left[\frac{1}{2}\right]^{-}+1 \cdot\left[\frac{1}{2}\right]^{+}+2 \cdot[0]+2 \cdot\left[t_{2}\right]^{-} .
\end{aligned}
$$

So in other words, everything properly assembles into the following $6 \mathrm{D}$ multiplets:

$$
\begin{aligned}
8 D & \rightarrow 7 D \rightarrow 6 D \\
S_{(3 / 2)}^{8 D} & \rightarrow S_{(3 / 2)}^{7 D} \rightarrow 2 \cdot S_{(3 / 2)}^{+}+2 \cdot S_{(3 / 2)}^{-} .
\end{aligned}
$$

\footnotetext{
${ }^{10}$ We thank D.S. Park for helpful discussions.
} 
With this caveat dealt with, we now see that we have correctly identified a 7D supermultiplet decoupled from the graviton:

$$
S_{(3 / 2)}^{7 D}=2 \cdot\left[\frac{3}{2}\right]+4 \cdot[1]+8 \cdot\left[\frac{1}{2}\right]+4 \cdot[0]+3 \cdot\left[t_{2}\right]+\left[t_{3}\right] .
$$

One might now ask whether it is actually consistent to consider such a multiplet decoupled from gravity. Indeed, the presence of the Rarita-Schwinger fields would seem to suggest that in any theory with a non-trivial S-matrix, gravity must indeed be included.

But if we have a trivial S-matrix (though a non-trivial theory!), we can consistently decouple gravity and simultaneously retain our gravitino multiplet. ${ }^{11}$ To illustrate, observe that when we compactify M-theory on a four-manifold, the action contains the following terms with the three-form potential:

$$
\operatorname{Vol}\left(M_{4}\right) \int_{7 D} d C_{(3)} \wedge * d C_{(3)}+\frac{1}{4 \pi i} \int_{M_{4}} \frac{G_{(4)}}{2 \pi} \int_{7 D} C_{(3)} \wedge d C_{(3)} .
$$

In the case where we decouple gravity, $\operatorname{Vol}\left(M_{4}\right)$ is quite large, so in this sense the three-form is also "decoupled". Additionally, however, we see that if there is a non-zero four-form flux, as can happen if we have M5-branes present, then there is a Chern-Simons-like coupling in seven dimensions. This places the three-form potential in a gapped phase and in particular means that the resulting supermultiplet is massive.

The fermions of the three-form multiplet decouple in a similar fashion. We note that in the 11D supergravity action, there is a coupling of the schematic form $\bar{\Psi}_{(3 / 2)} \cdot G_{(4)} \cdot \Psi_{(3 / 2)}$, so this flux also generates a mass for the gravitinos and Weyl fermions of $S_{(3 / 2)}^{7 D}$. The four zero-form and four one-form potentials all develop masses through the standard Stückelberg mechanism, and the kinetic terms:

$$
\left|d \phi_{(0)}+A_{(1)}\right|^{2}+\left|d A_{(1)}\right|^{2},
$$

with mass set by the compactification scale, just as for the three-form potential. The final set of terms involving the two-form potentials proceed in analogous fashion. Observe that the magnetic dual of the two-form is a three-form, so we can alternatively just write additional Chern-Simons like couplings to decouple these modes as well. All told, then, we see that the local excitations decouple, the only remnant in the $7 \mathrm{D}$ theory being a topological sector.

One can also consider coupling the $7 \mathrm{D}$ gravitino multiplet to the $7 \mathrm{D}$ vector multiplet. This proceeds, for example, through the topological term:

$$
\mu_{7 D} \int_{7 D} C_{(3)} \wedge \operatorname{Tr}(F \wedge F)
$$

where $F$ is the two-form field strength and $\mu_{7 D}$ is the "lift" of the $6 \mathrm{D}$ Green-Schwarz coupling [70, 85-87] to seven dimensions.

It is also possible to couple our supersymmetric theory to defects carrying propagating massless degrees of freedom. Observe that in this case, we need to impose suitable boundary conditions which will break half of the supersymmetry, but allow us to couple to appropriate supersymmetric edge modes.

\footnotetext{
${ }^{11}$ We thank M. Del Zotto for discussions on this point.
} 
In the above we have only sketched the main elements of the 7D supersymmetric theory. It would of course be most instructive to fill in these details in future work.

\section{$7 \quad$ IIB as an edge mode}

Motivated by the success of embedding our 7D topological theory in a supersymmetric compactification of M-theory, it is natural to ask whether a similar structure also holds for IIB string theory. Indeed, IIB supergravity contains a chiral four-form potential with self-dual field strength and there are notorious subtleties in writing an off-shell 10D action.

From our present perspective, the issue is in some sense forced once we demand proper quantization of fluxes in the quantum theory. In the special case where we restrict to level one for the chiral four-form (as happens for the standard IIB supergravity action) then we have an invertible theory in the sense of [33] so we expect to be able to decouple the bulk and boundary. Nevertheless, such an answer is rather unsatisfying because one could ask what happens at other "levels" of the chiral four-form, namely if we choose a different normalization for the two-point function. It is also disturbing that certain quantum questions cannot be easily accessed due to the absence of an off-shell action.

With this in mind, it is tempting to extend the standard 10D spacetime by an additional spatial direction to write an 11D topological theory of five-forms [31, 32], much as we already did in section 5 . We have already seen the utility of this perspective in our geometric unification of lower-dimensional topological theories.

Having gone to eleven dimensions, it is now irresistible to try and connect this with the 12D geometric formulation of F-theory [88]. Our aim in this section will be to present some suggestive - though conjectural - aspects of how to view all of IIB superstring theory as an edge mode of a bulk $10+2$-dimensional topological theory. ${ }^{12}$ In some sense this resurrects the original formulation of F-theory as genuinely living in $10+2$ dimensions rather than "merely" as a formal device for constructing non-perturbative $10 \mathrm{D}$ vacua. It also points the way to the construction of new self-consistent string vacua.

To accomplish this, we shall abandon from the start the notion of having propagating degrees of freedom in all twelve dimensions. Indeed, propagating degrees of freedom with two time directions leads to severe pathologies with causality. Rather, we shall immediately restrict attention to edge modes localized on a $9+1$-dimensional subspace. Our goal will be to see whether this can be fit together self-consistently.

To motivate our conjectural formulation of IIB as an edge mode, we shall proceed in a "bottom up" fashion, piecing together various consistency conditions. These will include:

(1) We attempt to quantize the IIB self-dual five-form flux by viewing the chiral four-form potential as an edge mode coupled to a bulk topological theory.

(2) We require the bulk theory to preserve supersymmetry, namely we must be able to assemble bulk supermultiplets.

(3) We must maintain the known $\mathrm{SL}(2, \mathbb{Z})$ covariance central to the formulation of IIB and its geometrization in F-theory.

\footnotetext{
${ }^{12}$ We thank C. Vafa for an inspirational discussion.
} 
Let us now proceed to enforce conditions (1)-(3). Along these lines, we briefly recall that the Ramond-Ramond sector of IIB string theory consists of a chiral four-form $c_{(4)}$, a two-form $c_{(2)}$, and a zero-form $c_{(0)}$. Continuing with our general philosophy, we shall at first attempt to lift this to an $11 \mathrm{D}$ space with $10 \mathrm{D}$ boundary. In our 11D space we introduce bulk higher-form potentials $C_{(5)}, C_{(3)}$ and $C_{(1)}$ so that the edge modes amount to degrees of freedom which cannot be gauged away, namely $C_{(p)}=d c_{(p-1)}$ on the boundary.

We immediately face an obstacle because the $11 \mathrm{D}$ supergravity spectrum in $10+1$ dimensions does not admit such degrees of freedom! There is, however, a loophole if we proceed to $10+2$ dimensions, namely ten spatial and two temporal dimensions. Supergravity multiplets in a $12 \mathrm{D}$ spacetime with this exotic signature have been considered previously, for example in references [89-96]. The key point is that in $10+2$ dimensions, one can impose a Majorana-Weyl condition on spinors which in turn allows for a match between bosonic and fermionic degrees of freedom. The supersymmetry algebra in $10+2$ dimensions has been studied in references [92, 95], and a proposed chiral supergravity theory has been constructed in [91, 94, 95] (for a different perspective, see e.g. [97, 98]). The general conclusion is that one ought to consider a theory with a four-form and two-forms, as well as a pair of null one-forms, $\mu_{+}$and $\mu_{-}$.

Before proceeding to how this matches with the form content we have, let us briefly explain how this earlier work makes contact with the more geometric formulation of $\mathrm{F}$ theory on an elliptically fibered Calabi-Yau with section which has become the de facto standard in modern practice. Along these lines, let us suppose that we have a standard F-theory background of the form $\mathbb{R}^{d-1,1} \times C Y_{n}$, where $C Y_{n}$ is an elliptically fibered CalabiYau $n$-fold with base $\mathcal{B}_{n-1}$ a Kähler manifold of complex dimension $(n-1)$ so that the total spacetime dimension is $10=d+2(n-1)$. The existence of a section means that there is an embedding of the $10 \mathrm{D}$ spacetime $\mathbb{R}^{d-1,1} \times \mathcal{B}_{n-1}$ in the $12 \mathrm{D}$ geometry. As such, we also have a local normal coordinate $z \in \mathcal{O}\left(-K_{\mathcal{B}_{n-1}}\right)$, and corresponding infinitessimal one-form $d z$. This $d z$ can in turn be decomposed into real and imaginary parts, and thus we get a pair of real one-forms, namely the Euclidean analogs of $\mu_{+}$and $\mu_{-}$presented above. Extending to the $12 \mathrm{D}$ total space, we can also construct a connection which parameterizes the breaking of $\mathrm{SO}(10,2)$ to $\mathrm{SO}(9,1)$ :

$$
\mu_{(1)}=\mu_{+} \cdot d \mu_{-} .
$$

Here, the "." refers to the contraction of one-form indices. ${ }^{13}$ This relation appears (in a slightly different form) in reference [99] (see also [95]), for example. ${ }^{14}$

Continuing in Euclidean signature for our two extra dimensions, the local geometry near the 10D spacetime is the Cartesian product of a cylinder $\mathcal{C}$ with the 10D spacetime. Topologically, $\mathcal{C}=S^{1} \times \mathbb{R}$. The local profile of the metric is in the conformal class:

$$
d s_{E u c}^{2}=d \theta^{2}+d r^{2},
$$

\footnotetext{
${ }^{13}$ For the perhaps more familiar case of breaking patterns of a compact Lie group, we would write $\mu_{(1)}=-i g^{\dagger} d g$, where $g$ is a generator of the group. Note that for a $\mathrm{U}(1)$ gauge theory with $g=\exp (i \theta)$, the formal one-form reduces to $d \theta$.

${ }^{14}$ In reference [95], the $\mathrm{SL}(2, \mathbb{Z})$ duality relation of IIB supergravity is imposed "by hand" at the classical $12 \mathrm{D}$ level, rather than as an emergent property from compactification on a $T^{2}$.
} 
so that $\theta$ is a periodic local coordinate, and $r$ parameterizes the radial profile of the cylinder. Globally, we of course complete this local presentation of the metric to construct an elliptic fibration over the 10D spacetime, with metric in the conformal class:

$$
d s_{E}^{2}=\left(d x+\tau\left(x_{10 D}\right) d y\right)\left(d x+\bar{\tau}\left(x_{10 D}\right) d y\right)
$$

where the complex structure of the elliptic curve is identified with the combination of IIB supergravity modes:

$$
\tau=c_{(0)}+i e^{-\phi}
$$

This elliptic fibration is central to the geometrization of $\operatorname{SL}(2, \mathbb{Z})$ duality in IIB string theory.

Let us now turn to the Lorentzian signature version, as dictated by supersymmetry in $10+2$ dimensions. We have already presented evidence that to properly quantize the 10D chiral four-form, we ought to extend our spacetime by one additional spatial direction, i.e., the $r$ coordinate of line (7.2). This means that if we insist on a supersymmetric theory in $(10,2)$ signature, we need to analytically continue $\theta \mapsto-i \theta$. So for us, the local profile of the metric in line (7.2) is instead:

$$
d s_{\text {Lor }}^{2}=-d \theta^{2}+d r^{2}
$$

The presence of an additional timelike direction means that a reduction along a null direction takes us from a $10+2$-dimensional spacetime to a $9+1$-dimensional spacetime.

At this point, something awkward appears to have happened. On the one hand, we have argued based on flux quantization considerations and supersymmetry that we need to add Lorentzian directions of signature $(1,1)$. On the other hand, the $\operatorname{SL}(2, \mathbb{Z})$ duality group of IIB is most naturally formulated in terms of a Euclidean $T^{2}$, and simply analytically continuing to Lorentzian signature fails to retain this structure. ${ }^{15}$ Again taking our cue from the original paper [88], we note that the Narain lattice for strings on a Euclidean $T^{2}$ has signature $(2,2)$ so it is not inconceivable that a $(1,1)$ signature spacetime could still replicate these features.

To put this on a firmer footing, we now observe that by construction, we do not allow any localized excitations in the two extra directions, this being the point of dealing with a bulk 12D topological theory in the first place. Nothing, however, disallows extended objects to wrap around compact cycles of the geometry. In particular, returning to our discussion of the Lorentzian cylinder geometry of line (7.5), we see that we can wrap an extended object along the timelike circle parameterized by the $\theta$ coordinate. The winding number along this circle has a Fourier transform which yields a dual periodic coordinate $\widetilde{\theta}$. From this perspective, there is again a Euclidean signature torus lurking, but in comparison with line (7.3), it will involve a dual coordinate $\widetilde{x}$ :

$$
d s_{\widetilde{E}}^{2}=\left(d \widetilde{x}+\tau\left(x_{10 D}\right) d y\right)\left(d \widetilde{x}+\bar{\tau}\left(x_{10 D}\right) d y\right) .
$$

\footnotetext{
${ }^{15}$ The main point is that it is not possible to define a family of Lorentzian signature torii in which the analog of $\tau$ smoothly varies. We thank D.R. Morrison for helpful comments on this point.
} 


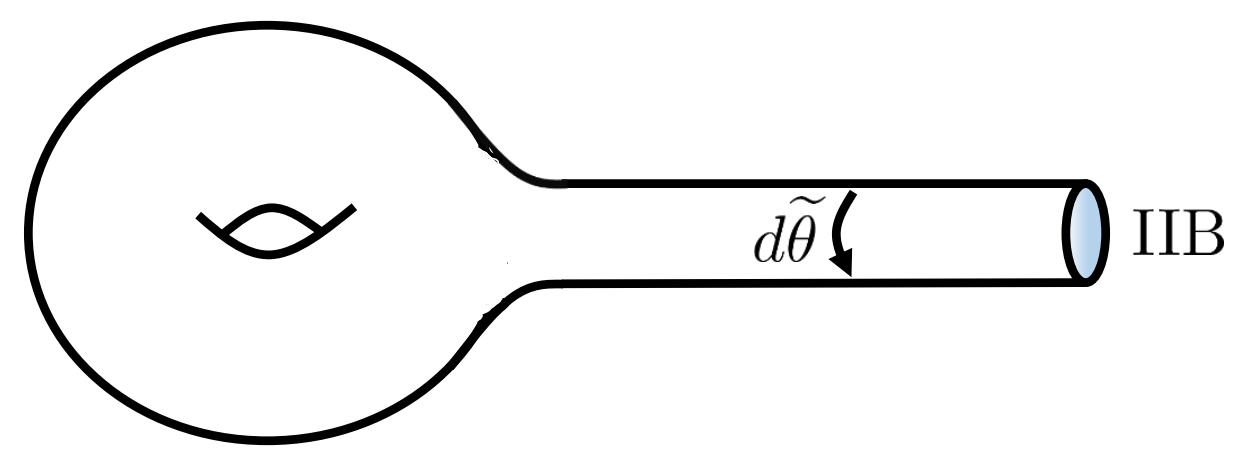

Figure 5. Depiction of the 12D geometry associated with F-theory and IIB as an edge mode in the special case where the axio-dilaton is constant. Here, we have passed from the $(10,2)$ signature spacetime to one in which a dual winding coordinate $\widetilde{\theta}$ appears. This takes us to a dualized spacetime of signature $(11,1)$ and in which the standard elliptic fiber of F-theory appears geometrically.

This sort of mixing of momentum and winding is a feature of geometric approaches to Tduality such as double field theory [100-102]. In some sense our discussion is less ambitious since we restrict to a topological sector from the start. We thus have in our extra $1+1$ dimensions a pair of null directions along which we can compactify on lightlike circles. Even though nothing propagates along them, extended objects can wrap the circles, and the consistent description of such degrees of freedom leads to a Euclidean signature torus, with complex structure the axio-dilaton of IIB strings. See figure 5 for a depiction of the geometry in the special case in which we have a constant elliptic fibration.

A satisfying byproduct of this proposal is that it helps to clarify the role of the elliptic fiber in M-theory/F-theory duality. Recall that in the standard picture, M-theory on the background $C Y_{n}$ is also described by F-theory on $S^{1} \times C Y_{n}$, where $C Y_{n}$ denotes the "same" elliptically fibered Calabi-Yau $n$-fold. In this process, one is supposed to collapse the Mtheory elliptic fiber to zero size to reach the F-theory limit. In the lower-dimensional context of IIA/IIB T-duality, one can consider similar limits, modulo the caveat that we interchange momentum and winding degrees of freedom. Here, we see that a similar consideration seems to hold, and in some sense is required to simultaneously satisfy our earlier conditions (1)-(3) mentioned at the beginning of this section. Precisely because we can interchangeably work in terms of either a $10+2$-dimensional spacetime, or one in which we trade a position coordinate for a winding coordinate to reach a dualized $11+1$ dimensional spacetime, we see that the procedure of dimensional reduction also carries over. In particular, a reduction of differential forms along the circle with coordinate $\tilde{\theta}$ proceeds as in a standard Kaluza-Klein compactification.

With the geometry of the spacetime dealt with, let us now return to our proposed mode content, now lifted to $10+2$ dimensions. We have so far identified a five-form, threeform and one-form, namely $C_{(5)}, C_{(3)}$, and $C_{(1)}$. Additionally, taking our cue from earlier work on supersymmetry in $10+2$ dimensions, we impose an anti-self-duality condition on the six-form field strength $F_{(6)}=-*_{12 D} F_{(6)}$. Given that our motivation was to properly quantize the fluxes of the IIB theory, and we have now introduced a self-duality constraint in $12 \mathrm{D}$, one might ask whether it is necessary to proceed up to 13 dimensions, perhaps 
along the lines of S-theory [103]. There is no reason to do so, at least from quantization considerations. The reason is that because of the presence of a distinguished null oneform, the way we read off the physical brane spectrum is different. Indeed, as we shortly explain, the physical degrees of freedom associated with our five-form potential require either contraction or wedging our five-form to a four-form or six-form, respectively. As such, the physical degrees of freedom are associated with a $2+2$-brane and $4+2$-brane, so we need not directly quantize the corresponding six-form flux units (since we have no $3+2$-branes to speak of). Indeed, turning the discussion around, we see that the absence of integral six-form fluxes actually eliminates the possibility of a $3+2$-brane, leaving us only with objects such as a $2+2$-brane or $4+2$-brane!

Comparing with the earlier supersymmetry literature in $10+2$ dimensions [89-96], we now ask how we can recover a four-form potential and a pair of two-form potentials, as well as the axio-dilaton. This all falls into place upon contracting our $p$-forms with the one-forms $\mu_{+}$and $\mu_{-}$, namely we write: ${ }^{16}$

$$
\mu_{ \pm} \cdot C_{(p)} \equiv C_{(p-1)}^{( \pm)} \cdot
$$

Note that this automatically generates covariant transformation rules for the two-form potentials and the scalars in the 10D IIB theory, and the anti-self-duality relation projects us onto an $\mathrm{SL}(2, \mathbb{Z})$ singlet for the chiral four-form potential.

A closely related point is that (as is well-known from M-/F-theory duality) the periods of our potentials along circles produces the expected IIB mode content. For example, the three-form reduces to the NS and RR two-forms, and transform as a doublet of $\mathrm{SL}(2, \mathbb{Z})$. The periods of the one-form potential $C_{(1)}$ along the two one-cycles produce a pair of scalars, and ratios of appropriate linear combinations reduces to the axio-dilaton $\tau$. Finally, the periods of the five-form are related by duality of its field strength, namely $F_{(6)}=-*_{12 D} F_{(6)}$, with $F_{(6)}=d C_{(5)}$, so the boundary four-form transforms as an $\mathrm{SL}(2, \mathbb{Z})$ singlet.

So far, we have focused on the RR sector, and have already seen a partial unification with some parts of the NS sector of the 10D boundary. To round out our discussion, we now turn to the 12D origin for the remaining NS degrees of freedom, namely the 10D metric. Since we have introduced explicit one-forms $\mu_{+}$and $\mu_{-}$, we expect the graviton to always have a formally infinite mass in two of the twelve directions. The gauge redundancy for a massless graviton:

$$
g_{A B} \rightarrow g_{A B}+\nabla_{A} C_{B}+\nabla_{B} C_{A},
$$

also means that we can alternatively package our mode content in terms of a 10D graviton, and a one-form $C_{(1)}$, the one introduced earlier from a bottom up perspective. By the same token, we expect a partially massive gravitino in $10+2$ dimensions to fill out the necessary fermionic degrees of freedom.

Let us now turn to the sense in which our system is actually in a gapped phase. Starting from the $11 \mathrm{D}$ action:

$$
\frac{1}{4 \pi i} \int_{11 D} C_{(5)} \wedge d C_{(5)},
$$

\footnotetext{
${ }^{16}$ Here, we are working in the $(11,1)$ signature geometry with a winding coordinate so as to make $\operatorname{SL}(2, \mathbb{Z})$ duality manifest.
} 
we can extend to 12D using our privileged one-form $\mu_{(1)}$ of line (7.1). By the same token, we can also introduce a related BF-like term to produce a mass gap for the one-form $C_{(1)}$ and its pairing to the three-form $C_{(3)}$. Writing out all proposed couplings to the higher-form potentials, we have:

$$
\begin{aligned}
S_{12 D, \text { top }}= & \frac{1}{4 \pi} \int_{12 D} \mu_{(1)} \wedge C_{(5)} \wedge d C_{(5)}+\frac{1}{4 \pi} \int_{12 D}\left(\mu_{(1)} \cdot C_{(3)}\right) \wedge d C_{(9)} \\
& +\frac{1}{4 \pi} \int_{12 D}\left(\mu_{(1)} \cdot C_{(5)}\right) \wedge G_{(4)} \wedge G_{(4)}
\end{aligned}
$$

where $G_{(4)}$ is the four-form field strength for the three-form potential, and we have used the magnetic dual of $C_{(1)}$, namely the nine-form $C_{(9)}$. The coupling of the second line has appeared in the F-theory literature before [97, 98, 104, 105]. Observe that we have dropped pre-factors of " $i$ " because we have two Lorentzian directions. Finally, note that the only appearance of the metric occurs in the privileged null direction via contraction with $\mu_{(1)}$. This dependence is sufficiently mild that we again obtain a trivial stress energy tensor, as required for a topological theory of this type. As far as we are aware, the couplings of line (7.10) have not been considered before.

We can also include a partially massive graviton, along the lines of reference [106]. Such theories have appeared in string theory and related holographic setups, see e.g. [107-109]. Let us note that in massive gravity, there are various concerns about superluminal propagation. Here, however, we are taking a rather different limit from what is typically discussed in phenomenological applications: for us, the mass in the decoupled directions is formally infinite, rather than small. An additional comment is that in the related context of warped compactification, a graviton zero mode can be localized on a lower-dimensional spacetime [110]. This is rather suggestive considering that in F-theory models, the base of the model has positive curvature, and thus the curvature is negative in the directions normal to the base inside the Calabi-Yau.

Returning to the topological couplings of our 12D action, note that in the $10 \mathrm{D}$ boundary, the first term reduces to a kinetic term for a chiral four-form, and the second term provides a Stückelberg mass for the RR and NS two-forms. Indeed, in F-theory backgrounds with non-trivial $\mathrm{SL}(2, \mathbb{Z})$ monodromy, these two-forms are generically projected out, receiving a mass due to such interactions. Line (7.11) reduces to the well-known IIB coupling $c_{(4)} \wedge h_{(3)}^{R R} \wedge h_{(3)}^{N S}$, which could in principle receive additional one-loop contributions from integrating out components of the massive gravitino.

It is also tempting to extend each stringy excitation of the $10 \mathrm{D}$ boundary by a $1+1$ dimensional direction in 12D, as for example in [92-96]. Following our topological route, we extend the F1- and D1-branes to a topological 2+2-brane which couples to the four-form obtained through the contraction $\mu_{(1)} \cdot C_{(5)}$. Some aspects of $2+2$-dimensional branes have recently been studied for example in [111], and for earlier work, see e.g. [112-116]. We can also extend a $3+1$-dimensional D3-brane to a $4+2$-dimensional topological brane which couples to the six-form $\mu_{(1)} \wedge C_{(5)}$. 
This also points the way to constructing new string vacua. For example, though we have for the most part concentrated on the case of unit coefficient in line (7.9), it is quite tempting to broaden our horizons to more general couplings of the form:

$$
\frac{N}{4 \pi i} \int_{11 D} C_{(5)} \wedge d C_{(5)} .
$$

There is a simple interpretation of this as winding modes along a timelike circle of the 12D geometry. Indeed, in an early speculative attempt to mimic the M-theory interpretation of D0-branes as momentum along a circle, reference [117] sought to interpret IIB D-instantons (namely $D_{-1}$-branes) in terms of "momentum" along a circle of a $12 \mathrm{D}$ spacetime. Here, we see that a background condensate of D-instantons produces a corresponding shift in the level of the five-form theory. In this formulation, we identify $N-1$ with a winding number for the metric, since the case of no D-instantons ought to correspond to the case of the "usual" IIB background with canonically normalized two-point function for the chiral four-form in ten dimensions. This provides a somewhat complementary motivation for IIB matrix models, and it would be interesting to revisit earlier proposals such as [118-120]. Departing one step further, it is tempting to consider $12 \mathrm{D}$ geometries without an elliptic fibration.

Apparently, then, the natural setting for many of our considerations resides in a $10+2-$ dimensional bulk topological theory. This appears to be compatible with the conditions of supersymmetry, $\mathrm{SL}(2, \mathbb{Z})$ duality, and also evades the pathologies of two-time physics and potential issues with massive gravity. We leave more detailed checks of this proposal for future work.

Summarizing, the 12D topological interpretation of superstrings is intriguing.

\section{Conclusions}

In this paper we have proposed a six-dimensional generalization of the fractional quantum Hall effect which makes use of the correspondence between a bulk 7D topological field theory and a $6 \mathrm{D}$ theory of edge modes. The bulk is given by a Chern-Simons like 7D theory of three-forms, and the edge mode theory is that of free anti-chiral two-forms. In the presence of a large background magnetic four-form flux, there is a direct analog with the usual fractional quantum Hall effect. We determined the leading order behavior of the analogous Laughlin wavefunction in various limits, and have also explained how this higher-dimensional starting point provides a unifying perspective on several lowerdimensional systems involving a bulk topological field theory coupled to edge modes. This 7D theory embeds in a limit of M-theory decoupled from gravity, and this in turn motivates a speculative conjecture on the interpretation of F-theory as a topological theory in $10+2$ dimensions coupled to $9+1$-dimensional edge modes associated with IIB strings. In the remainder of this section we discuss some avenues for future investigation.

There is by now a nearly complete list of supersymmetric 6D CFTs. One of the original motivations for this work was to better understand the topological structure of these fascinating theories. It is therefore quite tempting to ask whether we can develop a 
supersymmetric version of our analysis. For some discussion of a supersymmetric version of the fractional quantum Hall effect in two dimensions, see for example [121, 122].

In this work focused on the simplest situation where there is a single connected component to the boundary. In lower dimensions, it is often quite fruitful to consider additional disconnected components to the boundary. This in turn is intimately connected with the spectrum of non-local operators in the theory.

Another thread of our analysis is the unifying framework it provides for generating a rich class of lower-dimensional phenomena. We can already anticipate that various integer couplings can in many cases be recast as topological properties of a compactification manifold. It would be exciting to use this perspective to develop a systematic analysis of these possibilities.

One of the original motivations for this work was to better understand the topological sector of little string theories (for early constructions see e.g. [78, 123-128]), in which the pairing $\Omega_{I J}$ is no longer invertible since it has a zero eigenvalue $[39,129]$. Such little string theories are non-local, and thus not controlled by the standard axioms of local quantum field theory. It is natural to ask whether a suitable 7D theory exists with edge modes given by a little string theory.

We have also seen that our 7D topological theory admits a supersymmetric extension which embeds as a decoupling limit of the physical M-theory. Topological M-theory provides a unified framework for understanding various aspects of topological strings [130] (see also $[131,132])$. This theory is formulated in terms of an abelian three-form, but instead involves compactifying on a manifold of $G_{2}$ metric holonomy with action the integral of $C_{(3)} \wedge$ ${ }_{7 D} C_{(3)}$, where the metric is itself constructed from the associative three-form $[133,134]$. Adding such a term to a theory of a three-form gauge potential also leads to a mass gap. It would thus be interesting to compare the IR behavior of these two 7D theories.

Proceeding up to $10+2$ dimensions, we presented some tantalizing hints of an interpretation of F-theory as a bulk topological theory. Though quite conjectural, it already provides a novel starting point for realizing new classes of string vacua. It would clearly be very interesting to provide further evidence for this proposal. A particularly fascinating aspect of such a correspondence is the emergence of the IIB graviton as an edge mode excitation.

\section{Acknowledgments}

We thank F. Apruzzi, M. Del Zotto, T.T. Dumitrescu, O.J. Ganor, D.R. Morrison, D.S. Park and C. Vafa for helpful discussion. We also thank O.J. Ganor and D.R. Morrison for comments on an earlier draft. JJH thanks the 2016 and 2017 Summer workshops at the Simons Center for Geometry and Physics as well as the Aspen Center for Physics Winter Conference in 2017 on Superconformal Field Theories in $d \geq 4$, NSF grant PHY-1066293, for hospitality during part of this work. The work of JJH is supported by NSF CAREER grant PHY-1452037. LT thanks UNC Chapel Hill and the ITS at CUNY for hospitality during this work. The work of LT is supported by VR grant \#2014-5517 and by the "Geometry and Physics" grant from Knut and Alice Wallenberg Foundation. 
Open Access. This article is distributed under the terms of the Creative Commons Attribution License (CC-BY 4.0), which permits any use, distribution and reproduction in any medium, provided the original author(s) and source are credited.

\section{References}

[1] D.C. Tsui, H.L. Stormer and A.C. Gossard, Two-dimensional magnetotransport in the extreme quantum limit, Phys. Rev. Lett. 48 (1982) 1559 [inSPIRE].

[2] R.B. Laughlin, Anomalous quantum Hall effect: An incompressible quantum fluid with fractionallycharged excitations, Phys. Rev. Lett. 50 (1983) 1395 [inSPIRE].

[3] F.D.M. Haldane, Fractional quantization of the Hall effect: A Hierarchy of incompressible quantum fluid states, Phys. Rev. Lett. 51 (1983) 605 [INSPIRE].

[4] B.I. Halperin, Theory of the quantized Hall conductance, Helv. Phys. Acta 56 (1983) 75 [INSPIRE].

[5] B.I. Halperin, Statistics of quasiparticles and the hierarchy of fractional quantized Hall states, Phys. Rev. Lett. 52 (1984) 1583 [Erratum ibid. 52 (1984) 2390] [INSPIRE].

[6] J.K. Jain, Composite fermion approach for the fractional quantum Hall effect, Phys. Rev. Lett. 63 (1989) 199 [inSPIRE].

[7] S.M. Girvin and A.H. MacDonald, Off diagonal long range order, oblique confinement and the fractional quantum Hall effect, Phys. Rev. Lett. 58 (1987) 1252 [INSPIRE].

[8] X.G. Wen and A. Zee, Quantum Statistics and Superconductivity in Two Spatial Dimensions, Nucl. Phys. Proc. Suppl. 15 (1990) 135 [InSPIRE].

[9] B. Blok and X.G. Wen, Effective Theories of Fractional Quantum Hall Effect at Generic Filling Fractions, Phys. Rev. B 42 (1990) 8133 [INSPIRE].

[10] X.G. Wen and Q. Niu, Ground-state degeneracy of the fractional quantum Hall states in the presence of a random potential and on high-genus Riemann surfaces, Phys. Rev. B 41 (1990) 9377 [INSPIRE].

[11] Z.F. Ezawa and A. Iwazaki, Chern-Simons gauge theories for the fractional quantum Hall effect hierarchy and anyon superconductivity, Phys. Rev. B 43 (1991) 2637 [INSPIRE].

[12] X.G. Wen and A. Zee, Topological structures, universality classes, and statistics screening in the anyon superfluid, Phys. Rev. B 44 (1991) 274.

[13] J. Fröhlich and A. Zee, Large scale physics of the quantum Hall fluid, Nucl. Phys. B 364 (1991) 517 [INSPIRE].

[14] X.-G. Wen, Theory of the edge states in fractional quantum Hall effects, Int. J. Mod. Phys. B 6 (1992) 1711 [INSPIRE].

[15] A. Zee, Quantum Hall fluids, Lect. Notes Phys. 456 (1995) 99 [cond-mat/9501022] [INSPIRE].

[16] G.W. Moore and N. Read, Nonabelions in the fractional quantum Hall effect, Nucl. Phys. B 360 (1991) 362 [INSPIRE].

[17] D. Tong, Lectures on the Quantum Hall Effect, 2016, arXiv:1606.06687, http://inspirehep.net/record/1471556/files/arXiv:1606.06687.pdf [INSPIRE]. 
[18] S.-C. Zhang and J.-p. Hu, A Four-dimensional generalization of the quantum Hall effect, Science 294 (2001) 823 [cond-mat/0110572] [INSPIRE].

[19] J.H. Brodie, L. Susskind and N. Toumbas, How Bob Laughlin tamed the giant graviton from Taub-NUT space, JHEP 02 (2001) 003 [hep-th/0010105] [InSPIRE].

[20] B.A. Bernevig, C.-H. Chern, J.-P. Hu, N. Toumbas and S.-C. Zhang, Effective field theory description of the higher dimensional quantum Hall liquid, Annals Phys. 300 (2002) 185 [cond-mat/0206164] [INSPIRE].

[21] M. Fabinger, Higher dimensional quantum Hall effect in string theory, JHEP 05 (2002) 037 [hep-th/0201016] [INSPIRE].

[22] D. Karabali and V.P. Nair, Quantum Hall effect in higher dimensions, Nucl. Phys. B 641 (2002) 533 [hep-th/0203264] [INSPIRE].

[23] B.A. Bernevig, J.-p. Hu, N. Toumbas and S.-C. Zhang, The Eight-dimensional quantum Hall effect and the octonions, Phys. Rev. Lett. 91 (2003) 236803 [cond-mat/0306045] [INSPIRE].

[24] D. Karabali and V.P. Nair, The effective action for edge states in higher dimensional quantum Hall systems, Nucl. Phys. B 679 (2004) 427 [hep-th/0307281] [INSPIRE].

[25] K. Hasebe, Higher (Odd) Dimensional Quantum Hall Effect and Extended Dimensional Hierarchy, Nucl. Phys. B 920 (2017) 475 [arXiv:1612.05853] [INSPIRE].

[26] J. McGreevy, TASI 2015 Lectures on Quantum Matter (with a View Toward Holographic Duality), in proceedings of Theoretical Advanced Study Institute in Elementary Particle Physics: New Frontiers in Fields and Strings (TASI 2015), Boulder, CO, U.S.A., June 1-26, 2015, pp. 215-296 (2017) [DOI:10.1142/9789813149441_0004] [arXiv:1606.08953] [INSPIRE].

[27] E. Witten, AdS/CFT correspondence and topological field theory, JHEP 12 (1998) 012 [hep-th/9812012] [INSPIRE].

[28] O. Aharony and E. Witten, Anti-de Sitter space and the center of the gauge group, JHEP 11 (1998) 018 [hep-th/9807205] [INSPIRE].

[29] J.M. Maldacena, G.W. Moore and N. Seiberg, D-brane charges in five-brane backgrounds, JHEP 10 (2001) 005 [hep-th/0108152] [INSPIRE].

[30] E.P. Verlinde, Global aspects of electric-magnetic duality, Nucl. Phys. B 455 (1995) 211 [hep-th/9506011] [INSPIRE].

[31] D. Belov and G.W. Moore, Holographic Action for the Self-Dual Field, hep-th/0605038 [INSPIRE].

[32] D.M. Belov and G.W. Moore, Type II Actions from 11-Dimensional Chern-Simons Theories, hep-th/0611020 [inSPIRE].

[33] D.S. Freed and C. Teleman, Relative quantum field theory, Commun. Math. Phys. 326 (2014) 459 [arXiv:1212.1692] [INSPIRE].

[34] M. Del Zotto, J.J. Heckman, D.S. Park and T. Rudelius, On the Defect Group of a $6 D$ SCFT, Lett. Math. Phys. 106 (2016) 765 [arXiv:1503.04806] [INSPIRE].

[35] S. Monnier, The anomaly field theories of six-dimensional $(2,0)$ superconformal theories, arXiv:1706.01903 [INSPIRE]. 
[36] J.J. Heckman, D.R. Morrison and C. Vafa, On the Classification of 6D SCFTs and Generalized ADE Orbifolds, JHEP 05 (2014) 028 [Erratum ibid. 06 (2015) 017] [arXiv:1312.5746] [INSPIRE].

[37] M. Del Zotto, J.J. Heckman, A. Tomasiello and C. Vafa, 6d Conformal Matter, JHEP 02 (2015) 054 [arXiv: 1407.6359] [INSPIRE].

[38] J.J. Heckman, D.R. Morrison, T. Rudelius and C. Vafa, Atomic Classification of $6 D$ SCFTs, Fortsch. Phys. 63 (2015) 468 [arXiv:1502. 05405] [INSPIRE].

[39] L. Bhardwaj, Classification of $6 d \mathcal{N}=(1,0)$ gauge theories, JHEP 11 (2015) 002 [arXiv: 1502.06594] [INSPIRE].

[40] H.W.E. Jung, Darstellung der Funktionen eines algebraischen Körpers zweier unabhängiger Veränderlicher $x, y$ in der Umgebung einer Stelle $x=a, y=b, J$. Reine Angew. Math. 133 (1908) 289.

[41] F. Hirzebruch, Über vierdimensionale Riemannsche Flächen mehrdeutiger analytischer Funktionen von zwei komplexen Veränderlichen, Math. Ann. 126 (1953) 1.

[42] O. Riemenschneider, Deformationen von Quotientensingularitäten (nach zyklischen Gruppen), Math. Ann. 209 (1974) 211.

[43] S. Monnier, Topological field theories on manifolds with Wu structures, Rev. Math. Phys. 29 (2017) 1750015 [arXiv: 1607.01396] [INSPIRE].

[44] D. Belov and G.W. Moore, Classification of Abelian spin Chern-Simons theories, hep-th/0505235 [INSPIRE].

[45] A. Kapustin and N. Saulina, Topological boundary conditions in abelian Chern-Simons theory, Nucl. Phys. B 845 (2011) 393 [arXiv: 1008. 0654] [inSPIRE].

[46] B. de Wit, M. Lüscher and H. Nicolai, The Supermembrane Is Unstable, Nucl. Phys. B 320 (1989) 135 [INSPIRE].

[47] W. Taylor, The M(atrix) model of M-theory, NATO Sci. Ser. C 556 (2000) 91 [hep-th/0002016] [INSPIRE].

[48] E. Witten, On flux quantization in M-theory and the effective action, J. Geom. Phys. 22 (1997) 1 [hep-th/9609122] [INSPIRE].

[49] R. Floreanini and R. Jackiw, Selfdual Fields as Charge Density Solitons, Phys. Rev. Lett. 59 (1987) 1873 [INSPIRE].

[50] L. Álvarez-Gaumé and E. Witten, Gravitational Anomalies, Nucl. Phys. B 234 (1984) 269 [INSPIRE].

[51] O. Ganor and L. Motl, Equations of the $(2,0)$ theory and knitted five-branes, JHEP 05 (1998) 009 [hep-th/9803108] [INSPIRE].

[52] F. Bastianelli, S. Frolov and A.A. Tseytlin, Three point correlators of stress tensors in maximally supersymmetric conformal theories in $D=3$ and $D=6$, Nucl. Phys. B 578 (2000) 139 [hep-th/9911135] [INSPIRE].

[53] O.J. Ganor and J.L. Karczmarek, M(atrix) theory scattering in OM theory, JHEP 10 (2000) 024 [hep-th/0007166] [INSPIRE].

[54] O.J. Ganor, Six-dimensional tensionless strings in the large $N$ limit, Nucl. Phys. B 489 (1997) 95 [hep-th/9605201] [INSPIRE]. 
[55] D.S. Berman, M-theory branes and their interactions, Phys. Rept. 456 (2008) 89 [arXiv:0710.1707] [INSPIRE].

[56] P.-M. Ho and Y. Matsuo, M5 from M2, JHEP 06 (2008) 105 [arXiv:0804.3629] [INSPIRE].

[57] P.-M. Ho, Y. Imamura, Y. Matsuo and S. Shiba, M5-brane in three-form flux and multiple M2-branes, JHEP 08 (2008) 014 [arXiv: 0805.2898] [INSPIRE].

[58] Y. Nambu, Generalized Hamiltonian dynamics, Phys. Rev. D 7 (1973) 2405 [InSPIRE].

[59] N. Seiberg and E. Witten, String theory and noncommutative geometry, JHEP 09 (1999) 032 [hep-th/9908142] [INSPIRE].

[60] O. Aharony, O. Bergman, D.L. Jafferis and J. Maldacena, $\mathcal{N}=6$ superconformal Chern-Simons-matter theories, M2-branes and their gravity duals, JHEP 10 (2008) 091 [arXiv:0806.1218] [INSPIRE].

[61] O. Aharony, O. Bergman and D.L. Jafferis, Fractional M2-branes, JHEP 11 (2008) 043 [arXiv: 0807.4924] [INSPIRE].

[62] O.J. Ganor, Compactification of tensionless string theories, hep-th/9607092 [INSPIRE].

[63] E. Witten, Duality relations among topological effects in string theory, JHEP 05 (2000) 031 [hep-th/9912086] [INSPIRE].

[64] N. Seiberg and W. Taylor, Charge Lattices and Consistency of 6D Supergravity, JHEP 06 (2011) 001 [arXiv: 1103.0019] [inSPIRE].

[65] F. Apruzzi, F. Hassler, J.J. Heckman and I.V. Melnikov, From 6D SCFTs to Dynamic GLSMs, Phys. Rev. D 96 (2017) 066015 [arXiv:1610.00718] [InSPIRE].

[66] R. Penrose, Twistor algebra, J. Math. Phys. 8 (1967) 345 [inSPIRE].

[67] J.J. Heckman and H. Verlinde, Gravity Amplitudes from a Gaussian Matrix Model, JHEP 09 (2013) 150 [arXiv: 1112.5209] [INSPIRE].

[68] J.J. Heckman and H. Verlinde, Instantons, Twistors and Emergent Gravity, arXiv: 1112.5210 [INSPIRE].

[69] J. Heckman and H. Verlinde, Covariant non-commutative space-time, Nucl. Phys. B 894 (2015) 58 [arXiv: 1401.1810] [INSPIRE].

[70] D.R. Morrison and W. Taylor, Classifying bases for $6 D$ F-theory models, Central Eur. J. Phys. 10 (2012) 1072 [arXiv:1201.1943] [INSPIRE].

[71] F. Apruzzi, J.J. Heckman and T. Rudelius, Green-Schwarz Automorphisms and 6D SCFTs, JHEP 02 (2018) 157 [arXiv: 1707.06242] [INSPIRE].

[72] A. Vishwanath and T. Senthil, Physics of three dimensional bosonic topological insulators: Surface Deconfined Criticality and Quantized Magnetoelectric Effect, Phys. Rev. X 3 (2013) 011016 [arXiv: 1209.3058] [INSPIRE].

[73] O.J. Ganor, D.R. Morrison and N. Seiberg, Branes, Calabi-Yau spaces and toroidal compactification of the $\mathcal{N}=1$ six-dimensional $E_{8}$ theory, Nucl. Phys. B 487 (1997) 93 [hep-th/9610251] [INSPIRE].

[74] K.A. Intriligator, D.R. Morrison and N. Seiberg, Five-dimensional supersymmetric gauge theories and degenerations of Calabi-Yau spaces, Nucl. Phys. B 497 (1997) 56 [hep-th/9702198] [INSPIRE]. 
[75] M. Del Zotto, J.J. Heckman and D.R. Morrison, 6D SCFTs and Phases of $5 D$ Theories, JHEP 09 (2017) 147 [arXiv:1703.02981] [INSPIRE].

[76] S.M. Kravec and J. McGreevy, A gauge theory generalization of the fermion-doubling theorem, Phys. Rev. Lett. 111 (2013) 161603 [arXiv:1306.3992] [INSPIRE].

[77] D. Gaiotto, A. Kapustin, N. Seiberg and B. Willett, Generalized Global Symmetries, JHEP 02 (2015) 172 [arXiv: 1412.5148] [INSPIRE].

[78] E. Witten, Some comments on string dynamics, in Future perspectives in string theory. Proceedings, Conference, Strings'95, Los Angeles, U.S.A., March 13-18, 1995, pp. 501-523 (1995) [hep-th/9507121] [INSPIRE].

[79] A. Strominger, Open p-branes, Phys. Lett. B 383 (1996) 44 [hep-th/9512059] [InSPIRE].

[80] N. Seiberg, Nontrivial fixed points of the renormalization group in six-dimensions, Phys. Lett. B 390 (1997) 169 [hep-th/9609161] [INSPIRE].

[81] D. Gaiotto and S.S. Razamat, $\mathcal{N}=1$ theories of class $\mathcal{S}_{k}$, JHEP 07 (2015) 073 [arXiv: 1503.05159] [INSPIRE].

[82] J.J. Heckman, P. Jefferson, T. Rudelius and C. Vafa, Punctures for theories of class $\mathcal{S}_{\Gamma}$, JHEP 03 (2017) 171 [arXiv: 1609.01281] [INSPIRE].

[83] J.A. Strathdee, Extended Poincaré Supersymmetry, Int. J. Mod. Phys. A 2 (1987) 273 [INSPIRE].

[84] R. Blumenhagen, M. Fuchs and E. Plauschinn, The Asymmetric CFT Landscape in $D=4,6,8$ with Extended Supersymmetry, Fortsch. Phys. 65 (2017) 1700006 [arXiv: 1611.04617] [INSPIRE].

[85] M.B. Green, J.H. Schwarz and P.C. West, Anomaly Free Chiral Theories in Six-Dimensions, Nucl. Phys. B 254 (1985) 327 [INSPIRE].

[86] A. Sagnotti, A note on the Green-Schwarz mechanism in open string theories, Phys. Lett. B 294 (1992) 196 [hep-th/9210127] [INSPIRE].

[87] V. Sadov, Generalized Green-Schwarz mechanism in F-theory, Phys. Lett. B 388 (1996) 45 [hep-th/9606008] [INSPIRE].

[88] C. Vafa, Evidence for F-theory, Nucl. Phys. B 469 (1996) 403 [hep-th/9602022] [InSPIRE].

[89] L. Castellani, P. Fré, F. Giani, K. Pilch and P. van Nieuwenhuizen, Beyond $d=11$ Supergravity and Cartan Integrable Systems, Phys. Rev. D 26 (1982) 1481 [InSPIRE].

[90] E. Bergshoeff, M. de Roo and B. de Wit, Conformal Supergravity in Ten-dimensions, Nucl. Phys. B 217 (1983) 489 [INSPIRE].

[91] M.P. Blencowe and M.J. Duff, Supermembranes and the Signature of Space-time, Nucl. Phys. B 310 (1988) 387 [INSPIRE].

[92] I. Bars, Supersymmetry, p-brane duality and hidden space-time dimensions, Phys. Rev. D 54 (1996) 5203 [hep-th/9604139] [INSPIRE].

[93] S. Hewson and M. Perry, The twelve-dimensional super (2+2)-brane, Nucl. Phys. B 492 (1997) 249 [hep-th/9612008] [INSPIRE].

[94] H. Nishino, Supergravity in $(10+2)$-dimensions as consistent background for superstring, Phys. Lett. B 428 (1998) 85 [hep-th/9703214] [InSPIRE]. 
[95] H. Nishino, $N=2$ chiral supergravity in $(10+2)$-dimensions as consistent background for super (2+2)-brane, Phys. Lett. B 437 (1998) 303 [hep-th/9706148] [INSPIRE].

[96] S.F. Hewson, An Approach to F-theory, Nucl. Phys. B 534 (1998) 513 [hep-th/9712017] [INSPIRE].

[97] K.-S. Choi, Twelve-dimensional Effective Action and T-duality, Eur. Phys. J. C 75 (2015) 202 [arXiv: 1409.2476] [inSPIRE].

[98] K.-S. Choi, Supergravity in Twelve Dimension, JHEP 09 (2015) 101 [arXiv:1504.00602] [INSPIRE].

[99] J.H. Schwarz, Covariant Field Equations of Chiral $N=2$ D $=10$ Supergravity, Nucl. Phys. B 226 (1983) 269 [INSPIRE].

[100] W. Siegel, Superspace duality in low-energy superstrings, Phys. Rev. D 48 (1993) 2826 [hep-th/9305073] [INSPIRE].

[101] W. Siegel, Two vierbein formalism for string inspired axionic gravity, Phys. Rev. D 47 (1993) 5453 [hep-th/9302036] [INSPIRE].

[102] C. Hull and B. Zwiebach, Double Field Theory, JHEP 09 (2009) 099 [arXiv:0904.4664] [INSPIRE].

[103] I. Bars, S theory, Phys. Rev. D 55 (1997) 2373 [hep-th/9607112] [InSPIRE].

[104] S. Ferrara, R. Minasian and A. Sagnotti, Low-energy analysis of $M$ and $F$ theories on Calabi-Yau threefolds, Nucl. Phys. B 474 (1996) 323 [hep-th/9604097] [InSPIRE].

[105] R. Donagi and M. Wijnholt, Breaking GUT Groups in F-theory, Adv. Theor. Math. Phys. 15 (2011) 1523 [arXiv: 0808.2223] [INSPIRE].

[106] C. de Rham, G. Gabadadze and A.J. Tolley, Resummation of Massive Gravity, Phys. Rev. Lett. 106 (2011) 231101 [arXiv: 1011.1232] [INSPIRE].

[107] D. Vegh, Holography without translational symmetry, arXiv:1301.0537 [INSPIRE].

[108] M. Blake and D. Tong, Universal Resistivity from Holographic Massive Gravity, Phys. Rev. D 88 (2013) 106004 [arXiv:1308.4970] [INSPIRE].

[109] M. Blake, D. Tong and D. Vegh, Holographic Lattices Give the Graviton an Effective Mass, Phys. Rev. Lett. 112 (2014) 071602 [arXiv:1310.3832] [INSPIRE].

[110] A. Karch and L. Randall, Locally localized gravity, JHEP 05 (2001) 008 [hep-th/0011156] [INSPIRE].

[111] W.D. Linch and W. Siegel, Critical Super F-theories, arXiv:1507.01669 [INSPIRE].

[112] H. Ooguri and C. Vafa, Selfduality and $N=2$ String MAGIC, Mod. Phys. Lett. A 5 (1990) 1389 [INSPIRE].

[113] H. Ooguri and C. Vafa, Geometry of $\mathcal{N}=2$ strings, Nucl. Phys. B 361 (1991) 469 [INSPIRE].

[114] H. Ooguri and C. Vafa, $\mathcal{N}=2$ heterotic strings, Nucl. Phys. B 367 (1991) 83 [inSPIRE].

[115] D. Kutasov, E.J. Martinec and M. O'Loughlin, Vacua of M-theory and $N=2$ strings, Nucl. Phys. B 477 (1996) 675 [hep-th/9603116] [INSPIRE].

[116] D. Kutasov and E.J. Martinec, M-branes and $N=2$ strings, Class. Quant. Grav. 14 (1997) 2483 [hep-th/9612102] [INSPIRE]. 
[117] A.A. Tseytlin, Type IIB instanton as a wave in twelve-dimensions, Phys. Rev. Lett. 78 (1997) 1864 [hep-th/9612164] [INSPIRE].

[118] N. Ishibashi, H. Kawai, Y. Kitazawa and A. Tsuchiya, A Large $N$ reduced model as superstring, Nucl. Phys. B 498 (1997) 467 [hep-th/9612115] [INSPIRE].

[119] H. Aoki, S. Iso, H. Kawai, Y. Kitazawa and T. Tada, Space-time structures from IIB matrix model, Prog. Theor. Phys. 99 (1998) 713 [hep-th/9802085] [INSPIRE].

[120] H. Aoki, S. Iso, H. Kawai, Y. Kitazawa, A. Tsuchiya and T. Tada, IIB matrix model, Prog. Theor. Phys. Suppl. 134 (1999) 47 [hep-th/9908038] [inSPIRE].

[121] D. Tong and C. Turner, Quantum Hall effect in supersymmetric Chern-Simons theories, Phys. Rev. B 92 (2015) 235125 [arXiv:1508.00580] [INSPIRE].

[122] C. Vafa, Fractional Quantum Hall Effect and M-theory, arXiv:1511.03372 [INSPIRE].

[123] P.S. Aspinwall, Point-like instantons and the $\operatorname{Spin}(32) / \mathbb{Z}_{2}$ heterotic string, Nucl. Phys. B 496 (1997) 149 [hep-th/9612108] [INSPIRE].

[124] P.S. Aspinwall and D.R. Morrison, Point - like instantons on K3 orbifolds, Nucl. Phys. B 503 (1997) 533 [hep-th/9705104] [INSPIRE].

[125] N. Seiberg, New theories in six-dimensions and matrix description of M-theory on $T^{5}$ and $T^{5} / \mathbb{Z}_{2}$, Phys. Lett. B 408 (1997) 98 [hep-th/9705221] [INSPIRE].

[126] K.A. Intriligator, New string theories in six-dimensions via branes at orbifold singularities, Adv. Theor. Math. Phys. 1 (1998) 271 [hep-th/9708117] [INSPIRE].

[127] A. Hanany and A. Zaffaroni, Branes and six-dimensional supersymmetric theories, Nucl. Phys. B 529 (1998) 180 [hep-th/9712145] [INSPIRE].

[128] I. Brunner and A. Karch, Branes at orbifolds versus Hanany Witten in six-dimensions, JHEP 03 (1998) 003 [hep-th/9712143] [inSPIRE].

[129] L. Bhardwaj, M. Del Zotto, J.J. Heckman, D.R. Morrison, T. Rudelius and C. Vafa, F-theory and the Classification of Little Strings, Phys. Rev. D 93 (2016) 086002 [arXiv: 1511.05565] [INSPIRE].

[130] R. Dijkgraaf, S. Gukov, A. Neitzke and C. Vafa, Topological M-theory as unification of form theories of gravity, Adv. Theor. Math. Phys. 9 (2005) 603 [hep-th/0411073] [INSPIRE].

[131] A.A. Gerasimov and S.L. Shatashvili, Towards integrability of topological strings. I. Three-forms on Calabi-Yau manifolds, JHEP 11 (2004) 074 [hep-th/0409238] [INSPIRE].

[132] N. Nekrasov, A la recherche de la M-theorie perdue $Z$ theory: Chasing $M / F$-theory, in Annual International Conference on Strings, Theory and Applications (Strings 2004), Paris, France, 28 June-2 July, 2004 [hep-th/0412021] [INSPIRE].

[133] N.J. Hitchin, The Geometry of Three-Forms in Six Dimensions, J. Diff. Geom. 55 (2000) 547 [math/0010054] [INSPIRE].

[134] N.J. Hitchin, Stable forms and special metrics, math/0107101 [INSPIRE]. 